\title{
ÉQUIVALENCES RATIONNELLE ET NUMÉRIQUE SUR CERTAINES VARIÉTÉS DE TYPE ABÉLIEN SUR UN CORPS FINI
}

\author{
PAR BRUNO KAHN
}

RÉSUMÉ. - Nous prouvons que si $X$ est une variété projective lisse "de type abélien" sur un corps fini $k$ pour laquelle la conjecture de Tate est vérifiée (par exemple un produit de courbes elliptiques, d'après Spieß), l'équivalence rationnelle égale l'équivalence numérique sur $X$. La démonstration utilise des idées de Soulé, le théorème de semi-simplicité de Jannsen et un résultat d'Yves André et de l'auteur, inspiré par les résultats de S.I. Kimura sur les motifs de Chow de dimension finie. Nous en tirons quelques conséquences, parmi lesquelles : les conjectures de Lichtenbaum sont vraies pour $X$, le groupe de Chow de codimension 2 de $X$ est de type fini, la conjecture de Beilinson-Soulé vaut en poids $n$ pour le corps des fonctions $K$ de $X$ pourvu que $n \leqslant 2$ ou que $\operatorname{dim} X \leqslant 2$, la conjecture de Gersten vaut pour les anneaux de valuation discrète de corps résiduel $K$.

(c) 2003 Elsevier SAS

ABSTRACT. - We prove that if $X$ is a smooth projective variety $X$ "of abelian type" over a finite field $k$ for which the Tate conjecture holds (e.g. a product of elliptic curves [Math. Ann. 314 (1999) 285-290]), rational and numerical equivalences agree on $X$. The proof uses Soulé's ideas [Math. Ann. 268 (1984) $317-$ 345], U. Jannsen's semi-simplicity theorem [Invent. Math. 107 (1992) 447-452], and a result of Y. André and the author [Rend. Sem. Math. Univ. Padova 108 (2002) 107-291] inspired by S.I. Kimura's results on finite-dimensional Chow motives [J. Alg. Geom., à paraitre]. We give some consequences, among which: the conjectures of Lichtenbaum [Lect. Notes in Math., Vol. 1068, Springer, 1984, pp. 127-138, §7] hold true for $X$, the second Chow group of $X$ is finitely generated, the Beilinson-Soulé conjecture holds in weight $n$ for the function field of $X$ provided $n \leqslant 2$ or $\operatorname{dim} X \leqslant 2$, Gersten's conjecture holds for discrete valuation rings with residue field such a function field if $\operatorname{dim} X \leqslant 2$.

(C) 2003 Elsevier SAS

\section{Introduction}

Soient $k$ un corps fini de caractéristique $p$ et $G=\operatorname{Gal}(\bar{k} / k)$. Dans un article séminal [57, th. 4 i)], Soulé démontre que pour toute variété projective lisse $X$ de dimension $\leqslant 3$ et "de type abélien" (par exemple une variété abélienne ou un produit de courbes), l'application "classe de cycle $l$-adique"

$$
C H^{i}(X) \otimes \mathbf{Q}_{l} \rightarrow H^{2 i}\left(\bar{X}, \mathbf{Q}_{l}(i)\right)^{G}
$$

est bijective pour tout nombre premier $l \neq p$ et tout $i \geqslant 0$. Ceci est un cas particulier de deux conjectures fondamentales en géométrie arithmétique : la conjecture de Tate [61] (surjectivité 
de $(*)$ ) et la conjecture de Beilinson $[3,1.0]^{1}$ (injectivité de $(*)$ ). La démonstration consiste à considérer la décomposition motivique de $X$ pour se réduire au théorème de Tate sur les endomorphismes de variétés abéliennes [62] et à l'hypothèse de Riemann sur $k$ (Deligne [11]).

La conjecture de Tate est maintenant démontrée pour un bon nombre de variétés abéliennes sur $k$ (voir ci-dessous). La situation est différente pour la conjecture de Beilinson, qui n'est connue grosso modo qu'en codimension 1 (où elle est triviale); elle est d'ailleurs utilisée par Soulé dans ce cas pour obtenir $(*)$. Dans le présent article, nous utilisons une idée nouvelle de S.I. Kimura [39] pour la démontrer en toute codimension dans un certain nombre de cas nouveaux.

Plus précisément, notant $\mathcal{A}(k)=\mathcal{A}$ la catégorie des motifs de Chow sur $k$ à coefficients rationnels :

DÉfINITION 1. - a) Soit $B(k)$ l'ensemble des classes d'isomorphismes de $k$-variétés projectives lisses dont le motif de Chow est dans la sous-catégorie épaisse rigide $\mathcal{A}_{\mathrm{ab}}$ de $\mathcal{A}$ engendrée par les motifs d'Artin et les motifs de variétés abéliennes (ou de courbes, c'est la même chose).

On dit que $X$ est de type abélien si $X \in B(k)$.

b) Soit $B_{\text {tate }}(k) \subset B(k)$ le sous-ensemble des variétés $X$ vérifiant la conjecture de Tate (pour un nombre premier $l \neq p$ donné : cela ne dépend pas de $l, c f$. [62, Th. 2.9] puisque Frobenius opère de manière semi-simple sur la cohomologie $l$-adique de $X$, voir lemme 1.9 ci-dessous).

Exemples 1. - a) $B(k)$ contient l'ensemble $A(k)$ défini dans [57, 3.3.1].

b) $B_{\text {tate }}(k)$ contient les $X \in B(k)$ avec $\operatorname{dim} X \leqslant 3$ (Soulé, voir ci-dessus) et les produits de courbes elliptiques (Spiess [59]).

c) Milne a démontré que la conjecture de Hodge pour les variétés abéliennes complexes de type $C M$ implique la conjecture de Tate pour les variétés abéliennes sur $k$ [51]. Inconditionnellement, les résultats de [52] montrent qu'on peut prouver la conjecture de Tate pour "beaucoup" de variétés abéliennes $A$ sur $k$. C'est le cas si l'algèbre des "cycles de Tate" de $A$ est engendrée en degré 1, par réduction au théorème de Tate [62]. C'est ainsi que Spiess démontre la conjecture de Tate pour les produits de courbes elliptiques. D'autres exemples sont les puissances de variétés abéliennes simples "de type K3" de Zarhin [66] ou "presque ordinaires" de Lenstra et Zarhin [41], cf. [52, A.7, exemples]. Pour un exemple où cette condition n'est pas vérifiée mais où néanmoins la conjecture de Tate est démontrée, voir [52, ex. 1.8].

Dans cet esprit on a également l'important résultat suivant d'Yves André [1] : tout cycle de Tate sur une variété abélienne sur $k$ est "motivé".

d) Un exemple amusant de nature très légèrement différente est celui d'une hypersurface de Fermat

$$
X: X_{0}^{m}+\cdots+X_{d+1}^{m}=0
$$

où $m$ est tel que $q^{\nu} \equiv-1(\bmod m)$ pour un $\nu$ convenable (Tate, Katsura et Shioda, $\left.[61,37]\right)$.

THÉORÈME 1 ( $c f$. th. 1.10). - Pour tout $X \in B_{\text {tate }}(k)$, l'équivalence rationnelle est égale à l'équivalence numérique (à coefficients rationnels) sur $X$.

Dans [33], nous avons montré que la conjonction des conjectures de Tate et de Beilinson a des implications considérables. Dans cet esprit, nous tirons les conséquences suivantes du théorème 1 . Soient $X \in B_{\text {tate }}(k), d=\operatorname{dim} X$ et $K=k(X)$.

COROLlaIRE 1 (cor. 3.10). - Les conjectures de Lichtenbaum [43, §7] sont vraies pour X.

Corollaire 2 (th. 4.5). - Pour tout ouvert $U$ de $X$, les groupes $H^{0}\left(U, \mathcal{K}_{2}\right), H^{1}\left(U, \mathcal{K}_{2}\right)$ et $H^{2}\left(U, \mathcal{K}_{2}\right)=C H^{2}(U)$ sont de type fini. Le groupe $K_{3}(K)_{\text {ind }}$ est égal à $K_{3}(k)$.

\footnotetext{
${ }^{1}$ Mais aussi de Lichtenbaum en conjonction avec la conjecture de Tate, $\left.c f .[43, \S 7,7)\right]$.
} 
Pour obtenir les corollaires 1 et 2, nous prouvons des résultats plus précis faisant intervenir une nouvelle cohomologie introduite par Lichtenbaum [44] (corollaire 3.8).

Corollaire 3 (cor. 2.5). - La conjecture de Beilinson-Soulé est vraie en poids n pour K pourvu que $n \leqslant 2$ oи que $d \leqslant 2$.

À ma connaissance, ce sont les premiers exemples non triviaux (au-delà de la dimension 1) où cette conjecture est explicitement démontrée. Toutefois, pour $d=2$ ou pour $n=2, d=3$, les résultats de [57] suffiraient (voir [57, th. 4 iii)] et la démonstration du corollaire 2.5).

COROLlaire 4 (th. 4.6). - Si $d=2$, on a des isomorphismes canoniques pour tout $n \geqslant 0$

$$
\left(K_{n}^{M}(K) \oplus \bigoplus_{0 \leqslant i \leqslant n-1} H^{2 i-n-1}\left(K,(\mathbf{Q} / \mathbf{Z})^{\prime}(i)\right)\right) \otimes \mathbf{Z}_{(2)} \stackrel{\sim}{\longrightarrow} K_{n}(K) \otimes \mathbf{Z}_{(2)}
$$

où

$$
(\mathbf{Q} / \mathbf{Z})^{\prime}(i)=\underset{(m, \operatorname{car} k)=1}{\lim _{m}} \mu_{m}^{\otimes i} .
$$

Les facteurs $\otimes \mathbf{Z}_{(2)}$ proviennent du fait que nous utilisons la conjecture de Milnor prouvée par Voevodsky [64] (noter que l'homomorphisme lui-même n'est pas défini avant cette tensorisation); on pourrait les supprimer si la conjecture de Bloch-Kato était entièrement démontrée. Si l'on fait "tendre $k$ vers l'infini" dans le théorème 4 , on obtient une confirmation partielle d'une conjecture de Suslin [60, conj. 4.1 et note] : si un corps $F$ contient un corps algébriquement clos $F_{0}, K_{*}(F)$ est engendré multiplicativement par $K_{1}(F)$ et $K_{*}\left(F_{0}\right)$ (corollaire 4.7 et remarque 4.8). Ce résultat est faux en caractéristique zéro d'après de Jeu [27].

Corollaire 5 (cor. 2.6). - Supposons $d=2$, et soit $A$ un anneau de valuation discrète de corps des fractions $E$ et de corps résiduel K. Alors la conjecture de Gersten est vraie pour la $K$-théorie algébrique de $A$ : pour tout $n \geqslant 0$, la suite

$$
0 \rightarrow K_{n+1}(A) \rightarrow K_{n+1}(E) \rightarrow K_{n}(K) \rightarrow 0
$$

est exacte.

La démonstration du théorème 1 utilise trois ingrédients de manière essentielle : le fait que la conjecture de semi-simplicité est vraie pour la cohomologie $l$-adique de $X$, la semi-simplicité de la catégorie des motifs modulo l'équivalence numérique due à Jannsen [26] et un raffinement d'un résultat de Kimura [39, prop. 7.5] dû à André et à l'auteur [2, prop. 9.1.14]. La technique de démonstration, quant à elle, remonte à Soulé [57] via Geisser [16].

Le corollaire 1 se déduit du théorème 1 de manière relativement classique $[48,49]$; toutefois, l'introduction de la cohomologie de Lichtenbaum mentionnée ci-dessus simplifie bien les choses. Il faut un peu d'effort pour déduire la version "globale" des conjectures de Lichtenbaum de leur version localisée en chaque nombre premier : à cet égard, le lemme 3.9 est fort utile.

Le corollaire 3, quant à lui, se déduit assez facilement du résultat de Geisser selon lequel la conjugaison des conjectures de Tate et de Beilinson implique la conjecture de Parshin ( $c f$. corollaire 2.2).

La méthode de démonstration du théorème 1 et du corollaire 1 conduit à de nouvelles formulations des trois conjectures dont nous avons démontré l'équivalence dans [35, th. 3.4] : voir théorème 5.2.

Cet article est construit comme suit. Au $\S 1$, nous démontrons le théorème 1 . Dans les paragraphes suivants, nous "tirons les marrons du feu" : cette opération demande à l'occasion 
le port de gants ignifuges. Comme indiqué ci-dessus, les conséquences que nous donnons sont toutes des cas particuliers de conséquences des trois conjectures équivalentes de [35, th. 3.4], dont beaucoup ont été déjà dégagées dans [33] : nous nous sommes efforcé de rédiger les démonstrations de telle manière qu'elles puissent être reproduites telles quelles quand ces conjectures seront démontrées.

$\mathrm{Au} \S 2$ nous donnons les conséquences les plus immédiates du théorème 1 , dont les corollaires 3 et 5 . Au $\$ 3$, nous introduisons la cohomologie de Lichtenbaum (à coefficients dans les complexes de cycles de Bloch), étendons les classes de cycles motiviques $l$-adiques de [35] $(l \neq p=$ car $k)$ en des classes émanant de la cohomologie de Lichtenbaum motivique et construisons une classe de cycle $p$-adique correspondante; nous démontrons ensuite leur bijectivité pour $X \in B_{\text {tate }}(k)$. Nous en déduisons le corollaire 1 et des propriétés de finitude pour la cohomologie motivique de Lichtenbaum, meilleures que pour la cohomologie motivique étale (et conjecturées par Lichtenbaum dans [44, introduction] pour toute variété projective lisse). Nous en déduisons aussi une formule pour le conoyau de l'application "classe de cycle $l$-adique entière" $C H^{n}(X) \otimes \mathbf{Z}_{l} \rightarrow H_{\text {cont }}^{2 n}\left(X, \mathbf{Z}_{l}(n)\right)$ (corollaire 3.12). Enfin, dans le $\S 5$, nous investigons la mesure dans laquelle les méthodes de cet article pourraient rapprocher l'échéance de la démonstration des conjectures indiquées.

Une partie substantielle du travail de Kimura sur lequel nous nous appuyons a été obtenue indépendamment par Peter J. O'Sullivan (communication personnelle à André et à l'auteur) : notamment la notion de dimension finie au sens de Kimura (semi-positivité dans sa terminologie) et le fait que le motif de Chow d'une variété abélienne est de dimension finie au sens de Kimura. Il n'obtient par contre pas le théorème de nilpotence de Kimura, ni a fortiori la proposition 1.5.

Les lignes ci-dessus soulignent la dette que j' ai envers les travaux antérieurs apparaissant dans la bibliographie : ils sont trop nombreux pour être cités dans le détail. En particulier, il aurait sans doute été possible de rédiger les $\S \S 3-5$ en termes de cohomologie motivique étale sans le travail de Geisser [17], mais les résultats auraient été plus désagréables à énoncer et les démonstrations plus compliquées.

Je remercie Yves André, Thomas Geisser, Hélène Esnault, Ofer Gabber, Stephen Lichtenbaum, Christophe Soulé et Eckart Viehweg pour des commentaires pertinents sur la préparation de ce texte; en particulier T. Geisser pour avoir attiré mon attention sur le problème soulevé dans 3.2.1, et C. Soulé pour m'avoir encouragé à ne pas me limiter aux produits de courbes elliptiques. D'autre part, c'est avec Y. André que j'ai dégagé dans [2] la proposition 1.5 ci-dessous, résultat clé sur lequel repose tout ce travail. Enfin, je remercie le rapporteur pour des commentaires ayant permis d'améliorer l'exposition.

\section{1. Équivalence rationnelle et équivalence numérique}

Soient $\mathcal{A}$ la catégorie des motifs de Chow sur $k$ à coefficients rationnels et $\overline{\mathcal{A}}$ la catégorie des motifs purs sur $k$ modulo l'équivalence numérique, également à coefficients rationnels : cette dernière est abélienne semi-simple d'après [26]. On a un foncteur plein

$$
\begin{gathered}
\mathcal{A} \rightarrow \overline{\mathcal{A}}, \\
M \mapsto \bar{M} .
\end{gathered}
$$

Pour tout objet $M$ de $\mathcal{A}$ ou $\overline{\mathcal{A}}$, on note $F_{M}$ l'endomorphisme de Frobenius de $M$. Pour toute $k$-variété projective lisse $X$, on note $h(X)($ resp. $\bar{h}(X))$ le motif de $X$ dans $\mathcal{A}$ (resp. son image dans $\overline{\mathcal{A}})$.

Remarque 1.1. - Comme Voevodsky, nous adoptons la convention que le foncteur $X \mapsto h(X)$ est covariant, et non contravariant comme il est d'usage plus traditionnellement. Nous notons 
aussi $M \otimes L=M(1)$ au lieu de

$$
M \otimes L=M(-1),
$$

où $L$ est le motif de Lefschetz $\left(h\left(\mathbf{P}^{1}\right)=\mathbf{1} \oplus L\right)$. Avec ces conventions, on a

$$
C H^{n}(X) \otimes \mathbf{Q}=\mathcal{A}\left(h(X), L^{n}\right) \quad \text { et } \quad C H_{n}(X) \otimes \mathbf{Q}=\mathcal{A}\left(L^{n}, h(X)\right)
$$

pour toute $k$-variété projective lisse $X$, ainsi que $h(X)^{\vee}=h(X)(-\operatorname{dim} X)$.

Notation 1.2. - On note $\mathcal{A}_{\mathrm{ab}}$ la plus petite sous-catégorie épaisse (i.e. stable par sommes directes et facteurs directs) de $\mathcal{A}$ contenant les objets du type $h\left(A_{L}\right)$, où $L$ est une extension finie de $k$ et $A_{L}$ une variété abélienne sur $L$. Elle est rigide (stable par produit tensoriel et dual).

Rappelons la définition suivante [39] :

DÉFINITION 1.3. - Un objet $M \in \mathcal{A}$ est de dimension finie au sens de Kimura s'il existe une décomposition $M \simeq M_{+} \oplus M_{-}$et deux entiers $m, n \geqslant 0$ tels que $\Lambda^{m+1} M_{+}=\mathbf{S}^{n+1} M_{-}=0$.

On a :

THÉORÈME 1.4 (Kimura [39, th. 4.2, cor. 5.11, prop. 6.9]). - La sous-catégorie pleine $\mathcal{A}_{\mathrm{kim}}$ de $\mathcal{A}$ formée des motifs de dimension finie au sens de Kimura est épaisse et rigide. Elle contient $\mathcal{A}_{\mathrm{ab}}$.

La proposition suivante est directement inspirée de [39, prop. 7.5]. Sa preuve repose sur un calcul très technique qu'il est impossible de résumer ici ( $c f$. [2, prop. 7.2.7]).

Proposition 1.5 ([2, prop. 9.1.14]). - Soit $M \in \mathcal{A}_{\mathrm{kim}}$. Alors le noyau de

$$
\mathcal{A}(M, M) \rightarrow \overline{\mathcal{A}}(\bar{M}, \bar{M})
$$

est un idéal nilpotent.

Lemme 1.6. - Soit $N \in \mathcal{A}_{\mathrm{kim}}$. Supposons que $\bar{N}$ soit simple et que $F_{\bar{N}} \neq 1$. Alors

$$
\mathcal{A}(\mathbf{1}, N)=0 .
$$

Démonstration. - Soit $P \in \mathbf{Q}[T]$ le polynôme minimal de $F_{\bar{N}}$ : comme $\overline{\mathcal{A}}(\bar{N}, \bar{N})$ est une Qalgèbre à division, $P$ est un polynôme irréductible, différent de $T-1$ par hypothèse. D'après la proposition 1.5, il existe $n>0$ tel que $P\left(F_{N}\right)^{n}=0$. Soit $f \in \mathcal{A}(\mathbf{1}, N)$. Alors $F_{N} f=f$, d'où $P(1)^{n} f=0$ et $f=0$.

Proposition 1.7. - Soit $M \in \mathcal{A}_{\mathrm{kim}}$. Supposons que tout facteur simple $\bar{N}$ de $\bar{M}$ vérifie soit $\bar{N} \simeq \mathbf{1}$, soit $F_{\bar{N}} \neq 1$. Alors l'homomorphisme $\mathcal{A}(\mathbf{1}, M) \rightarrow \overline{\mathcal{A}}(\mathbf{1}, \bar{M})$ est un isomorphisme.

Démonstration. - En réappliquant la proposition 1.5, on voit que toute décomposition de $1_{\bar{M}}$ en somme d'idempotents orthogonaux se relève en une telle somme dans $\mathcal{A}(M, M)$. Pour cette décomposition, on a donc

$$
M=\bigoplus M_{i}
$$

où les $\bar{M}_{i}$ sont simples. De plus, en utilisant toujours la proposition 1.5 , on voit que

$$
\bar{M}_{i} \simeq \mathbf{1} \Rightarrow M_{i} \simeq \mathbf{1}
$$

La proposition 1.7 résulte donc de l'hypothèse et du lemme 1.6. 
Remarque 1.8. - Le principe de la démonstration du lemme 1.6 et de la proposition 1.7 est très proche dans l'esprit de [16, prop. 3.2].

Pour la commodité du lecteur, nous rappelons le lemme bien connu suivant. Soit $l$ un nombre premier différent de $p$ : on a un foncteur monoïdal

$$
H_{l}: \mathcal{A} \rightarrow V e c_{\mathbf{Q}_{l}}^{*}
$$

à valeurs dans les $\mathbf{Q}_{l}$-espaces vectoriels gradués, tel que $H_{l}(h(X))=H^{*}\left(\bar{X}, \mathbf{Q}_{l}\right)$ pour $X$ projective lisse.

Lemme 1.9. - Pour tout $M \in \mathcal{A}_{\mathrm{ab}}$, l'action de Frobenius sur $H_{l}(M)$ est semi-simple.

Démonstration. - On se ramène immédiatement au cas $M=h(A)$ où $A$ est une variété abélienne. L'assertion résulte alors du fait que "Frobenius arithmétique" est l'inverse de "Frobenius géométrique", de la semi-simplicité de l'algèbre $\operatorname{End}(A) \otimes \mathbf{Q}$ (Weil [65, p. 182, th. 28]) et du fait que "Frobenius géométrique" est dans le centre de cette algèbre.

ThÉORÈme 1.10. - Soient $X, X^{\prime}$ deux $k$-variétés projectives lisses telles que $X \times_{k} X^{\prime} \in$ $B_{\text {tate }}(k)$. Alors, pour tout $n \in \mathbf{Z}$, l'homomorphisme

$$
\begin{aligned}
& C H^{\operatorname{dim} X+n}\left(X^{\prime} \times X\right) \otimes \mathbf{Q}=\mathcal{A}\left(h\left(X^{\prime}\right), h(X)(n)\right) \\
& \rightarrow \overline{\mathcal{A}}\left(\bar{h}\left(X^{\prime}\right), \bar{h}(X)(n)\right)=A_{\mathrm{num}}^{\operatorname{dim} X+n}\left(X^{\prime} \times X\right) \otimes \mathbf{Q}
\end{aligned}
$$

est un isomorphisme.

Démonstration. - Par rigidité on se ramène à $X^{\prime}=\operatorname{Spec} k$. D'après le théorème 1.4 , $h(X)(n) \in \mathcal{A}_{\text {kim }}$. En tenant compte du lemme 1.9, on déduit de [63, th. 2.9] que l'équivalence homologique et l'équivalence numérique coïncident sur $X$. Il en résulte alors (cf. [50, prop. 2.6]) que pour tout facteur simple $\bar{N}$ de $\bar{h}(X)(n)$, on a soit $\bar{N} \simeq \mathbf{1}$ soit $F_{\bar{N}} \neq 1$. L'hypothèse de la proposition 1.7 est donc vérifiée, d'où le théorème 1.10 .

\section{Premières applications}

Corollaire 2.1. - Soit $X \in B_{\text {tate }}(k)$. Alors, pour tout $n$, l'ordre du pôle de la fonction zêta $\zeta(X, s)$ en $s=n$ est égal au rang de $C H^{n}(X)$ (qui est fini d'après le théorème 1.10).

Démonstration. - Un cas particulier du théorème 1.10 est que, sur $X$, l'équivalence homologique (relative à toute cohomologie de Weil) est égale à l'équivalence numérique. L'énoncé résulte donc du théorème 1.10 et de [63, th. 2.9].

La seconde application résulte de Geisser [16, th. 3.3] :

Corollaire 2.2. - Si $X \in B_{\text {tate }}(k)$, alors la conjecture de Beilinson-Parshin est vraie pour $X: K_{i}(X)$ est un groupe de torsion pour tout $i>0$.

(D'après loc. cit., la conclusion du corollaire résulte de la conjugaison des conjectures de Tate et de Beilinson pour $X$. Geisser démontre d'ailleurs ceci de manière analogue au théorème 1.10, en faisant opérer les correspondances algébriques sur $K_{*}(X) \otimes \mathbf{Q}$ par le théorème de RiemannRoch et en montrant que $K_{i}(S) \otimes \mathbf{Q}=0$ pour tout $i>0$ et tout facteur simple $S$ de $h(X) \stackrel{\sim}{\longrightarrow}$ $\bar{h}(X): c f$. aussi variante dans la démonstration de la proposition 3.1 ci-dessous. Bien entendu, c'est sa démonstration qui nous a inspiré...) 
Remarque 2.3. - Comme $\operatorname{dim} X \leqslant 1 \Rightarrow X \in B_{\text {tate }}(k)$, ceci redonne des théorèmes de Quillen [54] et Harder [23] par une voie entièrement différente. Noter que par contre on n'obtient pas ainsi la génération finie des groupes $K_{i}(X)$ [22] : à ce sujet, voir remarque 4.10.

Notons $H^{i}(X, \mathbf{Q}(n))$ les groupes de cohomologie motivique de $X$ à coefficients rationnels : dans cette section, ils peuvent être définis à la Beilinson comme espaces propres de groupes de $K$-théorie pour les opérations d'Adams [58]. Pour $X$ connexe on a

$$
H^{i}(X, \mathbf{Q}(0))= \begin{cases}\mathbf{Q} & \text { pour } i=0 \\ 0 & \text { pour } i \neq 0\end{cases}
$$

Rappelons la

Conjecture 2.4 (Beilinson-Soulé). - Pour tout corps $K$, on a $H^{i}(K, \mathbf{Q}(n))=0$ pour $n>0$ et $i \leqslant 0$.

Cette conjecture est connue pour les corps globaux et les corps de fonctions rationnelles en une variable sur un corps global.

Corollaire 2.5. - Soient $X \in B_{\text {tate }}(k), d=\operatorname{dim} X, K=k(X)$ et $n$ un entier $\geqslant 0$. Alors la conjecture 2.4 vaut pour $K$ en poids $n$, et on a même

$$
H^{i}(K, \mathbf{Q}(n))=0 \quad \text { pour } i \neq n
$$

dans les deux cas suivants :

(i) $d \leqslant 2$,

(ii) $n \leqslant 2$.

De plus, sous la condition (i), $K_{n}^{M}(K)$ et $K_{n}(K)$ sont de torsion première à $p$ pour $n \geqslant 3$ et $K_{n}^{M}(K)$ est de torsion impaire pour $n \geqslant 4$.

Démonstration. - Pour $i>n, c f$. Soulé [58, 2.8, cor. 1]. Pour le reste, $c f$. Geisser [16, th. 3.4] : on utilise le corollaire 2.2 et la suite spectrale de coniveau pour la cohomologie motivique. Si $d \leqslant 2$, les corps résiduels $F$ de $X$ autres que $K$ sont de dimension 0 ou 1 , donc les énoncés " $H^{i}(F, \mathbf{Q}(n))=0$ pour $i<n$ " et " $H^{i}(F, \mathbf{Q}(n))=0 \operatorname{pour} n>\operatorname{dim} F$ " résultent de la remarque 2.3. Si $n \leqslant 2$, les poids intervenant dans les termes $E_{1}^{p, q}$ avec $p>0$ sont $\leqslant 1$, et l'énoncé est encore connu (Kratzer [40, cor. 6.8]). L'assertion "première à $p$ " résulte de Geisser et Levine [18] (voir aussi Izhboldin [24] pour $K_{*}^{M}(K)$ ), et la dernière assertion pour car $k \neq 2$ résulte de la conjecture de Milnor (Voevodsky [64]).

Corollaire 2.6. - Soit $A$ un anneau de valuation discrète de corps des fractions $E$ et de corps résiduel $K$, où $K=k(X)$ avec $X \in B_{\text {tate }}(k), \operatorname{dim} X \leqslant 2$. Alors, pour tout $n \geqslant 0$, la suite

$$
0 \rightarrow K_{n+1}(A) \rightarrow K_{n+1}(E) \rightarrow K_{n}(K) \rightarrow 0
$$

(extraite de la suite exacte de localisation de Quillen) est exacte.

Démonstration. - Soit $m>0$ premier à $p=$ car $k$. Le diagramme commutatif au signe près

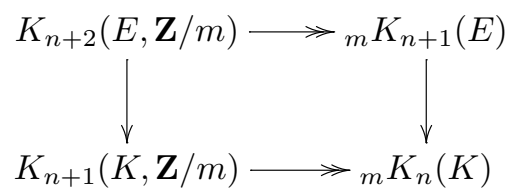


et la conjecture de Gersten pour $K_{*}(-, \mathbf{Z} / m)$ (Gillet [21]) montrent que l'homomorphisme résidu ${ }_{m} K_{n+1}(E) \rightarrow{ }_{m} K_{n}(K)$ est surjectif. L'assertion résulte donc du corollaire 2.5 pour $n \geqslant 3$, et est bien connue pour $n \leqslant 2$ puisqu' alors $K_{n}(K)=K_{n}^{M}(K)$.

\section{Classes de cycle motiviques}

\subsection{Cohomologie motivique}

Nous définirons la cohomologie motivique d'une $k$-variété lisse $X$ comme l'hypercohomologie de Zariski $H_{\text {Zar }}^{i}(X, \mathbf{Z}(n))$ des complexes de cycles de Bloch décalés ( $c f$. [5,7,19,35] pour les détails). Nous aurons aussi besoin de considérer l'hypercohomologie de ces complexes par rapport à d'autres topologies, et de les comparer. En particulier, le résultat ci-dessous sera d'usage constant :

Proposition 3.1 ([35, prop. 1.18]). - Pour toute variété lisse X, les homomorphismes

$$
H_{\text {Zar }}^{i}(X, \mathbf{Q}(n)) \rightarrow H_{\text {èt }}^{i}(X, \mathbf{Q}(n))
$$

sont des isomorphismes.

On a aussi :

Proposition 3.2. - Soit $X \in B_{\text {tate }}(k)$. Alors $H_{\mathrm{Zar}}^{i}(X, \mathbf{Q}(n))=0$ pour $i \neq 2 n$.

Démonstration. - Cela résulte du corollaire 2.2 et du fait que $H_{\mathrm{Zar}}^{i}(X, \mathbf{Q}(n)) \simeq g r_{\gamma}^{n} K_{2 n-i}(X)$ [5]. (Variante : raisonner directement comme dans la démonstration du théorème 1.10, en utilisant le fait que Frobenius agit sur ce groupe par multiplication par $q^{n}, c f .[16]$.)

\subsection{Cohomologie de Lichtenbaum}

Nous allons utiliser la cohomologie introduite par Lichtenbaum dans [44]. ${ }^{2}$ Cette cohomologie a été également étudiée par Geisser [17].

Rappelons [43, prop. 2.2] que la catégorie des faisceaux pour la topologie de Lichtenbaum sur une $k$-variété $X$ de type fini est équivalente à la catégorie des faisceaux étales $\mathbf{Z}$-équivariants sur $\bar{X}=X \times_{k} \bar{k}$, où l'action de $\mathbf{Z}$ sur $\bar{X}$ est donnée par le morphisme de Frobenius galoisien. Pour un tel faisceau $\mathcal{F}$, on pose

$$
H_{W}^{0}(X, \mathcal{F})=H_{\text {ét }}^{0}(\bar{X}, \mathcal{F})^{\mathbf{Z}}
$$

et la cohomologie de Lichtenbaum $H_{W}^{*}$ est définie comme les foncteurs dérivés de

$$
\mathcal{F} \mapsto H_{W}^{0}(X, \mathcal{F}) .
$$

En particulier on a une suite spectrale [44, prop. 2.3]

$$
H^{p}\left(\mathbf{Z}, H_{\text {ét }}^{q}(\bar{X}, C)\right) \Rightarrow H_{W}^{p+q}(X, C)
$$

pour tout complexe de faisceaux étales $\mathbf{Z}$-équivariants.

\footnotetext{
${ }^{2}$ Lichtenbaum dénomme cette cohomologie Weil-étale cohomology. Il nous paraît plus pratique et plus juste de la rebaptiser cohomologie de Lichtenbaum, la terminologie "cohomologie de Weil" pouvant de toute façon prêter à confusion. 
Pour comparer cohomologie étale et cohomologie de Lichtenbaum, le point de vue de Geisser $[17, \S 2]$ est d'identifier la catégorie des faisceaux étales sur $X$ à la catégorie des faisceaux étales $\hat{\mathbf{Z}}$-équivariants (topologiques discrets) sur $\bar{X}$, où $\hat{\mathbf{Z}}=\operatorname{Gal}(\bar{k} / k)$ [12, Exp. XIII, 1.1.3]. Soit $\mathcal{T}_{\mathbf{Z}}(X)\left(\right.$ resp. $\left.\mathcal{T}_{\hat{\mathbf{Z}}}(X)\right)$ la catégorie des $\bar{X}$-faisceaux étales $\mathbf{Z}$-équivariants (resp. $\hat{\mathbf{Z}}$-équivariants) : on a des foncteurs adjoints évidents

$$
\gamma_{X}^{*}: \mathcal{T}_{\hat{\mathbf{Z}}}(X) \rightarrow \mathcal{T}_{\mathbf{Z}}(X), \quad\left(\gamma_{X}\right)_{*}: \mathcal{T}_{\mathbf{Z}}(X) \rightarrow \mathcal{T}_{\hat{\mathbf{Z}}}(X)
$$

Notons $\mathcal{T}_{\mathbf{Z}}$ et $\mathcal{T}_{\hat{\mathbf{Z}}}$ les catégories de faisceaux correspondantes sur les "grands sites lisses" correspondant à la catégorie $S m / k$ des $k$-variétés lisses de type fini. On a des foncteurs adjoints correspondants

$$
\gamma^{*}: \mathcal{T}_{\hat{\mathbf{z}}} \rightarrow \mathcal{T}_{\mathbf{Z}}, \quad \gamma_{*}: \mathcal{T}_{\mathbf{Z}} \rightarrow \mathcal{T}_{\hat{\mathbf{z}}}
$$

induisant des foncteurs adjoints sur les catégories dérivées

$$
\gamma^{*}: D\left(\mathcal{T}_{\hat{\mathbf{Z}}}\right) \rightarrow D\left(\mathcal{T}_{\mathbf{Z}}\right), \quad R \gamma_{*}: D\left(\mathcal{T}_{\mathbf{Z}}\right) \rightarrow D\left(\mathcal{T}_{\hat{\mathbf{Z}}}\right)
$$

\subsubsection{Mise en garde}

Le foncteur $R \gamma_{*}$ n'est pas conservatif. Par exemple, soit $\mathbf{Q}\langle n\rangle$ le $\mathbf{Z}[\mathbf{Z}]$-module de support $\mathbf{Q}$, l'action du générateur de $\mathbf{Z}$ étant donnée par $r \mapsto q^{n} r$. Alors $\mathbf{Q}\langle n\rangle$ définit un objet de $\mathcal{T}_{\mathbf{Z}}$, mais on a $H_{W}^{*}(X, \mathbf{Q}\langle n\rangle)=0$ pour tout $X \in S m / k$ dès que $n \neq 0:$ c'est immédiat à partir de (3.1).

Pour obvier cet inconvénient, introduisons la sous-catégorie épaisse $D_{\text {in }}\left(\mathcal{T}_{\mathbf{Z}}\right)$ de $D\left(\mathcal{T}_{\mathbf{Z}}\right)$ formée des complexes $C$ tels que $H_{W}^{*}(X, C)=0$ pour tout $X \in S m / k$, et notons

$$
\bar{D}\left(\mathcal{T}_{\mathbf{Z}}\right)=D\left(\mathcal{T}_{\mathbf{Z}}\right) / D_{\text {in }}\left(\mathcal{T}_{\mathbf{Z}}\right)
$$

Soit $\bar{\gamma}^{*}$ le composé de $\gamma^{*}$ avec le foncteur de localisation $D\left(\mathcal{T}_{\mathbf{Z}}\right) \rightarrow \bar{D}\left(\mathcal{T}_{\mathbf{Z}}\right)$. Alors $R \gamma_{*}$ se factorise en un foncteur exact

$$
\bar{R} \gamma_{*}: \bar{D}\left(\mathcal{T}_{\mathbf{Z}}\right) \rightarrow D\left(\mathcal{T}_{\hat{\mathbf{Z}}}\right)
$$

qui est conservatif par construction, et adjoint à droite de $\bar{\gamma}^{*}$. Nous aurons besoin de $\bar{R} \gamma_{*}$ plutôt que de $R \gamma_{*}$ pour une formulation correcte du théorème 5.2 (iv).

\subsection{Cohomologie de Lichtenbaum motivique}

Nous considérerons principalement la cohomologie de Lichtenbaum à coefficients dans les complexes de cycles de Bloch. Pour tout $X \in S m / k$, le foncteur $R\left(\gamma_{X}\right)_{*}$ induit des homomorphismes canoniques

$$
H_{\text {ét }}^{i}(X, \mathbf{Z}(n)) \rightarrow H_{W}^{i}(X, \mathbf{Z}(n)) .
$$

Le résultat suivant de Geisser sera d'usage constant : notons $\beta$ le Bockstein et $e$ le générateur de $H_{W}^{1}(k, \mathbf{Z})=\operatorname{Hom}(\mathbf{Z}, \mathbf{Z})$ envoyant (par exemple) le Frobenius géométrique sur 1 . On a de longues suites exactes $[17$, th. 6.1$]$

$$
\begin{aligned}
\cdots & \rightarrow H_{\text {êt }}^{i}(X, \mathbf{Z}(n)) \rightarrow H_{W}^{i}(X, \mathbf{Z}(n)) \\
& \rightarrow H_{\text {ét }}^{i-1}(X, \mathbf{Z}(n)) \otimes \mathbf{Q} \stackrel{\partial}{\rightarrow} H_{\text {ét }}^{i+1}(X, \mathbf{Z}(n)) \rightarrow \cdots
\end{aligned}
$$

où $\partial$ est donnée par la composition 


$$
\begin{aligned}
& H_{\text {ét }}^{i-1}(X, \mathbf{Z}(n)) \otimes \mathbf{Q}=H_{\text {ét }}^{i-1}(X, \mathbf{Q}(n)) \rightarrow H_{\text {ét }}^{i-1}(X, \mathbf{Q} / \mathbf{Z}(n)) \\
& \stackrel{\cdot e}{\rightarrow} H_{\text {ét }}^{i}(X, \mathbf{Q} / \mathbf{Z}(n)) \stackrel{\beta}{\rightarrow} H_{\text {ét }}^{i+1}(X, \mathbf{Z}(n))
\end{aligned}
$$

(cf. [33, prop. 9.12]).

Rappelons que d'après Geisser et Levine [19, th. 1.5] et [18, th. 8.3], on a des isomorphismes canoniques $H_{\text {êt }}^{i}(X, \mathbf{Q} / \mathbf{Z}(n)) \simeq \bigoplus_{l} H_{\text {ét }}^{i}\left(X, \mathbf{Q}_{l} / \mathbf{Z}_{l}(n)\right)$, où la somme porte sur tous les nombres premiers et

$$
H_{\text {ét }}^{i}\left(X, \mathbf{Q}_{l} / \mathbf{Z}_{l}(n)\right)= \begin{cases}H_{\text {ét }}^{i}\left(X, \lim _{l^{s}}^{\otimes n}\right) & \text { pour } l \neq p, \\ H_{\text {ét }}^{i-n}\left(X, \underline{\lim } \nu_{s}(n)\right) & \text { pour } l=p,\end{cases}
$$

où $\mu_{l^{s}}^{\otimes n}$ est le faisceau des racines $l^{s}$-ièmes de l'unité tordues et $\nu_{s}(n)$ est le $n$-ième faisceau de Hodge-Witt logarithmique de niveau $s$.

On a aussi :

Proposition 3.3 ([17, th. 6.5 et 6.7]). - a) Pour toute $k$-variété lisse $X$ de dimension $d$ et pour tout $n \geqslant 0$, on a $H_{W}^{i}(X, \mathbf{Z}(n))=0$ pour $i>\sup (2 d+1, n+d+1)$.

b) $\mathrm{Si} X$ est de plus projective, on a un diagramme commutatif

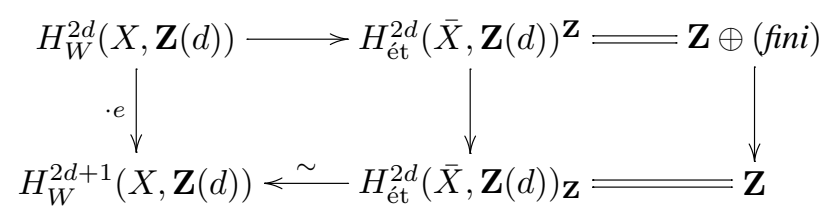

où l'isomorphisme $H_{\text {ét }}^{2 d}(\bar{X}, \mathbf{Z}(d))_{\mathbf{Z}}=\mathbf{Z}$ provient de l'homomorphisme "degré"

$$
H_{\text {ét }}^{2 d}(\bar{X}, \mathbf{Z}(d)) \simeq C H^{d}(\bar{X}) \rightarrow \mathbf{Z}
$$

(cf. [17, dém. du lemme 6.4 a)]).

Nous aurons aussi besoin de la

Proposition 3.4. - Pour toute variété lisse $X, H_{W}^{i}(X, \mathbf{Z}(n))$ est de torsion pour $i>2 n+1$.

Démonstration. - Cela résulte par exemple du fait que $H_{\mathrm{Zar}}^{i}(\bar{X}, \mathbf{Q}(n))=H_{\text {ét }}^{i}(\bar{X}, \mathbf{Q}(n))=0$ pour $i>2 n$ et de la suite spectrale (3.1).

\subsection{Classe de cycle $l$-adique}

Fixons un nombre premier $l \neq p$. Nous allons utiliser une application classe de cycle motivique l-adique pour la cohomologie de Lichtenbaum. Cela revient à étendre les homomorphismes de $[35, \S 1.4]$

$$
H_{\text {êt }}^{i}(X, \mathbf{Z}(n)) \otimes \mathbf{Z}_{l} \rightarrow H_{\text {cont }}^{i}\left(X, \mathbf{Z}_{l}(n)\right)
$$

en des homomorphismes provenant de $H_{W}^{i}(X, \mathbf{Z}(n)) \otimes \mathbf{Z}_{l}$.

(Rappelons que le groupe de droite est défini dans [35] comme l'hypercohomologie de l'objet $\mathbf{Z}_{l}(n)^{c}:=R \lim \mu_{l^{s}}^{\otimes n}$ de la catégorie dérivée des faisceaux abéliens sur $X_{\text {ét }}:$ ce n'est autre que la cohomologie étale continue de Jannsen [25]. Le corps de base $k$ étant fini, les théorèmes de finitude standard impliquent que l'homomorphisme naturel

$$
H_{\text {cont }}^{i}\left(X, \mathbf{Z}_{l}(n)\right) \rightarrow \lim _{\longleftarrow} H_{\text {ét }}^{i}\left(X, \mu_{l^{\nu}}^{\otimes n}\right)
$$

$4^{\mathrm{e}}$ SÉRIE - TOME $36-2003-\mathrm{N}^{\circ} 6$ 
est un isomorphisme : ce groupe coïncide donc avec celui considéré par exemple dans [9].)

Indiquons rapidement comment on procède : notons $\alpha:(S m / k)_{\text {ét }} \rightarrow(S m / k)_{\text {Zar }}$ le foncteur de projection entre les sites définis par la catégorie $S m / k$ des $k$-variétés lisses de type fini munie respectivement des topologies étale et de Zariski. Dans [35, th. 1.17], nous avons défini un objet $\mathbf{Z}(n) \in D^{-}\left(A b\left((S m / k)_{\text {Zar }}\right)\right)$ dont la restriction à toute $k$-variété lisse $X$ est quasi-isomorphe au complexe de cycles de Bloch de poids $n$ sur $X$, puis un morphisme dans la catégorie dérivée $[35,(1.8)]$

$$
\alpha^{*} \mathbf{Z}(n) \stackrel{L}{\otimes} \mathbf{Z}_{l} \rightarrow \mathbf{Z}_{l}(n)_{\text {ét }}^{c}
$$

où $\mathbf{Z}_{l}(n)_{\text {ét }}^{c}=R \lim _{l^{\nu}}^{\otimes n}$ dans la catégorie dérivée des faisceaux étales, que nous identifions canoniquement à $D\left(\mathcal{T}_{\hat{\mathbf{Z}}}\right)(c f .3 .2)$.

Définissons de même $\mathbf{Z}_{l}(n)_{W}^{c}=R \lim ^{*} \gamma^{*} \mu_{l^{\nu}}^{\otimes n}$ dans $D\left(\mathcal{T}_{\mathbf{Z}}\right)$ (ibid.). On a alors un morphisme composé

$$
\gamma^{*} \alpha^{*} \mathbf{Z}(n) \rightarrow \gamma^{*} \mathbf{Z}_{l}(n)_{\text {ét }}^{c} \rightarrow \mathbf{Z}_{l}(n)_{W}^{c} .
$$

C'est le morphisme cherché : il induit des homomorphismes

$$
H_{W}^{i}(X, \mathbf{Z}(n)) \otimes \mathbf{Z}_{l} \rightarrow H_{W}^{i}\left(X, \mathbf{Z}_{l}(n)_{W}^{c}\right)
$$

Il reste à remarquer que l'adjoint $\mathbf{Z}_{l}(n)_{\text {ét }}^{c} \rightarrow R \gamma_{*} \mathbf{Z}_{l}(n)_{W}^{c}$ du morphisme

$$
\gamma^{*} \mathbf{Z}_{l}(n)_{\text {ét }}^{c} \rightarrow \mathbf{Z}_{l}(n)_{W}^{c}
$$

est un quasi-isomorphisme : cela résulte du fait que $R \gamma_{*}$ et $R \lim$ commutent et de l'analogue de (3.2) à coefficients $\mu_{l^{\nu}}^{\otimes n}$, qui montre que $\mu_{l^{\nu}}^{\otimes n} \rightarrow R \gamma_{*} \gamma^{*} \mu_{l^{\nu}}^{\otimes n} \longleftarrow$ est un quasi-isomorphisme pour tout $n$ (cf. [17, cor. 3.4]). On en conclut que l'homomorphisme canonique

$$
H_{\mathrm{cont}}^{i}\left(X, \mathbf{Z}_{l}(n)\right) \rightarrow H_{W}^{i}\left(X, \mathbf{Z}_{l}(n)_{W}^{c}\right)
$$

est bijectif, et on en déduit bien l'application "classe de cycle" promise :

$$
H_{W}^{i}(X, \mathbf{Z}(n)) \otimes \mathbf{Z}_{l} \rightarrow H_{\text {cont }}^{i}\left(X, \mathbf{Z}_{l}(n)\right) .
$$

(Bien entendu, on pourrait aussi procéder naïvement à partir de $[19, \S 3.7]$, qui est de toute façon à la base de notre construction, voir aussi $[6, \S 4]$, en définissant (3.5) "variété par variété" et en évitant [35, (1.8)], mais les détails seraient plus pénibles à rédiger.)

En fait :

LEMME 3.5. - L'homomorphisme (3.5) n'est autre que celui induit par le morphisme $\Phi_{n}: \alpha^{*} \mathbf{Z}(n) \otimes^{L} \mathbf{Z}_{l}(0)_{\text {ét }}^{c} \rightarrow \mathbf{Z}_{l}(n)_{\text {ét }}^{c} d e$ [35, conj. 3.2].

Démonstration. - Rappelons que $\Phi_{n}$ est défini comme la composition

$$
\alpha^{*} \mathbf{Z}(n) \stackrel{L}{\otimes} \mathbf{Z}_{l}(0)_{\text {ét }}^{c} \rightarrow \mathbf{Z}_{l}(n)_{\text {ét }}^{c} \stackrel{L}{\otimes} \mathbf{Z}_{l}(0)_{\text {ét }}^{c} \rightarrow \mathbf{Z}_{l}(n)_{\text {ét }}^{c}
$$

où la première flèche se déduit de (3.3) et la suivante est donnée par le cup-produit. Nous allons voir que $\Phi_{n}$ se déduit de (3.4) par application du foncteur $R \gamma_{*}$. 
En effet, on a déjà vu le quasi-isomorphisme $\mathbf{Z}_{l}(n)_{\text {ét }}^{c} \stackrel{\sim}{\longrightarrow} R \gamma_{*} \mathbf{Z}_{l}(n)_{W}^{c}$. D'autre part, d'après $[17$, th. 3.3], le morphisme de double adjonction

$$
R \gamma_{*} \mathbf{Z}^{L} \otimes \alpha^{*} \mathbf{Z}(n) \rightarrow R \gamma_{*} \gamma^{*} \alpha^{*} \mathbf{Z}(n)
$$

est également un quasi-isomorphisme. Dans [33, th. 4.6 et 6.3 , nous avons établi un quasiisomorphisme

$$
\mathbf{Z}_{l}(0)_{\text {ét }}^{c} \simeq \mathbf{Z}^{c} \otimes \mathbf{Z}_{l}
$$

où $\mathbf{Z}^{c}$ est un certain complexe de longueur 1. Enfin, dans [17, th. 4.2], Geisser établit un quasiisomorphisme $\mathbf{Z}^{c} \simeq R \gamma_{*} \mathbf{Z}$. L'assertion résulte aisément de tous ces quasi-isomorphismes, en suivant leurs définitions respectives.

\subsection{Classe de cycle $p$-adique}

Posons

$$
\mathbf{Z}_{p}(n)_{\text {ét }}^{c}=R \lim _{\longleftarrow} \nu_{r}(n)[-n] \quad(n \geqslant 0)
$$

où $\nu_{r}(n)$ est le $n$-ième faisceau de Hodge-Witt logarithmique de niveau $r$, et de même

$$
\mathbf{Z}_{p}(n)_{W}^{c}=R \underset{\lim }{\longleftarrow} \gamma^{*} \nu_{r}(n)[-n] .
$$

Pour $n<0$, on pose $\mathbf{Z}_{p}(n)_{\text {ét }}^{c}=0$ et $\mathbf{Z}_{p}(n)_{W}^{c}=0$.

En passant à la limite sur la construction de [18, dém. du th. 8.3], on obtient une classe de cycle motivique $p$-adique pour la cohomologie étale

$$
\alpha^{*} \mathbf{Z}(n) \otimes \mathbf{Z}_{p} \rightarrow \mathbf{Z}_{p}(n)_{\text {ét }}^{c}
$$

puis une classe de cycle motivique $p$-adique pour la cohomologie de Lichtenbaum

$$
\gamma^{*} \alpha^{*} \mathbf{Z}(n) \otimes \mathbf{Z}_{p} \rightarrow \gamma^{*} \mathbf{Z}_{p}(n)_{\text {ét }}^{c} \rightarrow \mathbf{Z}_{p}(n)_{W}^{c}
$$

et plus concrètement, pour une variété lisse $X$ donnée, des homomorphismes

$$
H_{W}^{i}(X, \mathbf{Z}(n)) \otimes \mathbf{Z}_{p} \rightarrow H_{\text {cont }}^{i}\left(X, \mathbf{Z}_{p}(n)\right)
$$

où $H_{\text {cont }}^{i}\left(X, \mathbf{Z}_{p}(n)\right):=H_{\text {ét }}^{i}\left(X, \mathbf{Z}_{p}(n)_{\text {ét }}^{c}\right) \stackrel{\sim}{\longrightarrow} H_{W}^{i}\left(X, \mathbf{Z}_{p}(n)_{W}^{c}\right)(c f .3 .4)$.

\subsection{Bijectivité des classes de cycle}

ThÉORÈmE 3.6. - Si $X \in B_{\text {tate }}(k)$, (3.5) est un isomorphisme pour tous $i, n$ et tout $l \neq p$, et (3.8) est un isomorphisme pour tous $i, n$.

Démonstration. - Traitons d'abord le cas de (3.5). D'après le lemme 3.5, il suffit de voir que le morphisme induit par $\Phi_{n}$ est un isomorphisme pour tout $n$; cela résulte du théorème 1.10 , du corollaire 2.1 et de [35, th. 3.4]. (On pourrait aussi procéder directement à partir de la conjecture de Tate et du théorème 1.10, mais ce serait plus laborieux.) On en déduit déjà : 
LEMmE 3.7. - Pour $X \in B_{\text {tate }}(k)$, on a $H_{W}^{i}(X, \mathbf{Q}(n))=0$ pour $i<2 n$ et la composition

$$
C H^{n}(X) \otimes \mathbf{Q} \simeq H_{\text {Zar }}^{2 n}(X, \mathbf{Q}(n)) \rightarrow H_{W}^{2 n}(X, \mathbf{Q}(n))
$$

est un isomorphisme pour tout $n \geqslant 0$.

Démonstration. - Le premier point résulte par récurrence sur $i$ de la suite exacte (3.2) (cf. propositions 3.1 et 3.2). Cette même suite exacte implique alors que

$$
H_{\text {ét }}^{2 n}(X, \mathbf{Q}(n)) \rightarrow H_{W}^{2 n}(X, \mathbf{Q}(n))
$$

est un isomorphisme, et on conclut par la proposition 3.4.

Démontrons maintenant que (3.8) est aussi un isomorphisme. On procède directement, en imitant la méthode de $[35, \S 3.4]$ : on remarque d'abord que la version de (3.8) à coefficients $\mathbf{Q}_{p} / \mathbf{Z}_{p}$ est un isomorphisme d'après [la faisceautisation étale de] [18, th. 8.3] (ce fait est vrai pour toute $k$-variété lisse). Par le lemme des 5 , on se ramène à démontrer que (3.8) $\otimes \mathbf{Q}$ est un isomorphisme.

On sait déjà que $H_{W}^{i}(X, \mathbf{Q}(n))=0$ pour $i<2 n$ (par le lemme 3.7) et pour $i>2 n+1$ (par la proposition 3.4). La même chose est vraie pour les groupes $H_{\text {cont }}^{i}\left(X, \mathbf{Q}_{p}(n)\right)$, en utilisant un théorème de Gabber [9, th. 3$]$ et la suite exacte longue

$$
\begin{aligned}
\cdots & \rightarrow H_{\text {ét }}^{i-n-1}\left(X, \nu_{\infty}(n)\right) \rightarrow H_{\text {cont }}^{i}\left(X, \mathbf{Z}_{p}(n)\right) \\
& \rightarrow H_{\text {cont }}^{i}\left(X, \mathbf{Q}_{p}(n)\right) \rightarrow H_{\text {ét }}^{i-n}\left(X, \nu_{\infty}(n)\right) \rightarrow \cdots .
\end{aligned}
$$

Il reste donc à traiter les cas $i=2 n$ et $i=2 n+1$. Milne [48, prop. 5.4] a démontré qu'on peut utiliser l'action galoisienne sur les groupes $H^{i}\left(\bar{X}, \mathbf{Q}_{p}(n)\right)$ à la place de l'action de Frobenius sur la cohomologie cristalline pour calculer la fonction $\zeta(X, s)$. En particulier, en tenant compte de l'hypothèse de Riemann [11] et de [38], cela démontre que

$$
H^{i}\left(\bar{X}, \mathbf{Q}_{p}(n)\right)^{G}=H^{i}\left(\bar{X}, \mathbf{Q}_{p}(n)\right)_{G}=0 \quad \text { pour } i \neq 2 n
$$

(cf. [9]). Considérons le diagramme commutatif ( $c f$. [35, dém. de la prop. 3.9])

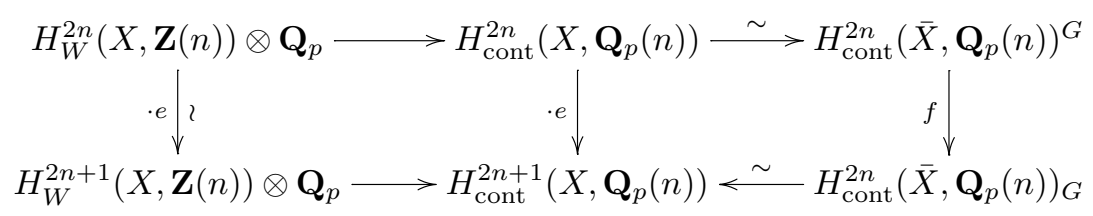

où $f$ est la composition

$$
H_{\text {cont }}^{2 n}\left(\bar{X}, \mathbf{Q}_{p}(n)\right)^{G} \hookrightarrow H_{\text {cont }}^{2 n}\left(\bar{X}, \mathbf{Q}_{p}(n)\right) \longrightarrow H_{\text {cont }}^{2 n}\left(\bar{X}, \mathbf{Q}_{p}(n)\right)_{G}
$$

(voir [48, prop. 6.5] pour la commutativité du carré de droite). La flèche verticale de gauche est un isomorphisme par le cas $l \neq p$, et les flèches horizontales de droite sont des isomorphismes grâce à (3.9).

En procédant comme dans $[63, \S 2]$ et en utilisant [48, prop. 5.4] et [38], on voit que le corollaire 2.1 implique que $f$ est bijective et que la composition

$$
C H^{n}(X) \otimes \mathbf{Q}_{p} \rightarrow H_{W}^{2 n}(X, \mathbf{Z}(n)) \otimes \mathbf{Q}_{p} \rightarrow H_{\text {cont }}^{2 n}\left(\bar{X}, \mathbf{Q}_{p}(n)\right)^{G}
$$


est surjective. De plus, le théorème 1.10 implique que cette composition est injective. En utilisant le lemme 3.7, on en déduit que la composition horizontale supérieure dans (3.10) est bijective. Il en résulte que tous les homomorphismes de (3.10) sont bijectifs.

\subsection{Conséquences}

Corollaire 3.8. - Si $X \in B_{\text {tate }}(k)$ et $d=\operatorname{dim} X$,

a) L'accouplement

$$
H_{W}^{2 n}(X, \mathbf{Z}(n)) \times H_{W}^{2 d-2 n}(X, \mathbf{Z}(d-n)) \rightarrow H_{W}^{2 d}(X, \mathbf{Z}(d)) \rightarrow \mathbf{Z}
$$

est non dégénéré modulo torsion pour tout $n$.

b) Pour tout $(i, n)$, l'accouplement

$$
H_{W}^{i}(X, \mathbf{Z}(n))_{\text {tors }} \times H_{W}^{2 d+1-i}(X, \mathbf{Q} / \mathbf{Z}(d-n)) \rightarrow H_{W}^{2 d+1}(X, \mathbf{Q} / \mathbf{Z}(d)) \stackrel{\sim}{\longrightarrow} \mathbf{Q} / \mathbf{Z}
$$

induit un accouplement parfait de groupes finis

$$
H_{W}^{i}(X, \mathbf{Z}(n))_{\text {tors }} \times H_{W}^{2 d+2-i}(X, \mathbf{Z}(d-n))_{\text {tors }} \rightarrow \mathbf{Q} / \mathbf{Z} .
$$

c) (cf. [44, introduction]) Les groupes $H_{W}^{i}(X, \mathbf{Z}(n))$ sont de type fini pour tout $i$, finis pour $i \notin\{2 n, 2 n+1\}$ ou pour $n>d$ et nuls pour $i \leqslant 0($ si $n>0)$.

d) Le noyau et le conoyau du cup-produit par e

$$
H_{W}^{2 n}(X, \mathbf{Z}(n)) \rightarrow H_{W}^{2 n+1}(X, \mathbf{Z}(n))
$$

sont finis.

e) L'homomorphisme canonique

$$
H_{\text {ét }}^{i}(X, \mathbf{Z}(n)) \rightarrow H_{W}^{i}(X, \mathbf{Z}(n))
$$

est un isomorphisme pour $i \leqslant 2 n$.

f) Pour tout $n \in \mathbf{Z}$, on a $H_{W}^{i}(X, \mathbf{Z}(n))=0$ pour $i>2 d+1$.

Démonstration. - Il résulte de [11,38,15] que $H_{\text {cont }}^{i}\left(X, \mathbf{Z}_{l}(n)\right)_{\text {tors }}$ est fini pour tout $i \in \mathbf{Z}, \mathbf{y}$ compris pour $l=p$, et nul pour presque tout $l(c f$. [9, th. 2 et 3$])$. De plus, $H_{\text {cont }}^{i}\left(X, \mathbf{Z}_{l}(n)\right)$ est fini pour $i \neq 2 n, 2 n+1$ ou pour $n>d$ (ibid.). Il résulte d'abord de ceci et du théorème 3.6 que c) est vrai pour $i \notin\{2 n, 2 n+1\}$ ou $n>d$, et est vrai pour $i \in\{2 n, 2 n+1\}$ après tensorisation par $\mathbf{Z}_{l}$ (pour tout $l$ ).

L'assertion b) est vraie après tensorisation par $\mathbf{Z}_{l}$ par le cas de la cohomologie étale continue ( $c f$. [48, th. 1.14] pour le cas $l=p$ ); sa version entière en résulte directement. De même, a) est vrai après tensorisation par $\mathbf{Z}_{l}$. La version entière de a) et de $c$ ) résulte alors du lemme suivant, que nous n'avons pas trouvé dans la littérature (cf.toutefois [47, Ch. V, §3, Lemma 3.26]) :

LEMme 3.9. - Soient $R$ un anneau commutatif et $A \times B \rightarrow R$ un accouplement de deux $R$-modules plats $A, B$.

a) Supposons que cet accouplement devienne non dégénéré après tensorisation par $R_{l}$ pour un idéal premier l de $R$, où $R_{l}$ désigne le complété de $R$ en $l$. Alors il est non dégénéré.

b) Supposons $R$ intègre noethérien et soit $K$ son corps des fractions. Si, de plus, $A \otimes K$ ou $B \otimes K$ est un $K$-espace vectoriel de dimension finie, alors $A$ et $B$ sont de type fini. 
Démonstration. - a) Considérons l'homomorphisme

$$
A \rightarrow \operatorname{Hom}(B, R)
$$

Notons $A_{l}:=A \otimes_{R} R_{l}$, etc. On a une chaîne d'homomorphismes

$$
A_{l} \rightarrow \operatorname{Hom}(B, R)_{l} \rightarrow \operatorname{Hom}\left(B_{l}, R_{l}\right)
$$

où la composition est injective par hypothèse. Par conséquent, le premier homomorphisme est lui aussi injectif. Comme $A$ est plat, $A \rightarrow A_{l}$ est injectif, donc (3.12) est aussi injectif. On procède de même pour montrer la non-dégénérescence de l'autre côté.

b) Pour fixer les idées, supposons que $\operatorname{dim}_{K} B \otimes K<\infty$. Soit $\left(b_{1}, \ldots, b_{n}\right)$ une famille d'éléments de $B$ qui définit une base de $B \otimes K$, et soit $B^{\prime}$ le sous-module de $B$ engendré par les $b_{i}$. Comme pour tout $b \in B$, il existe $r \in R$ tel que $r b \in B^{\prime}$, l'application

$$
\operatorname{Hom}(B, R) \rightarrow \operatorname{Hom}\left(B^{\prime}, R\right)
$$

est injective. Donc $A$ s'injecte dans un $R$-module de type fini et il est donc lui-même de type fini. En échangeant $A$ et $B$, on obtient la même conclusion pour $B$.

Revenons à la démonstration du corollaire $3.8:$ il reste à démontrer d), e) et f). L'assertion d) résulte de c) et du fait qu'elle est vraie après tensorisation par $\mathbf{Q}$, comme il résulte de la démonstration du théorème 3.6. D'autre part e) découle de c) et de (3.2), par récurrence sur $i$. Pour voir $\mathrm{f}$ ), on peut supposer $n>d$ d'après la proposition $3.3 \mathrm{a}$ ). En réutilisant c) et (3.2), on obtient une chaîne d'isomorphismes

$$
H_{\text {ét }}^{i-1}(X, \mathbf{Q} / \mathbf{Z}(n)) \stackrel{\sim}{\longrightarrow} H_{\text {ét }}^{i}(X, \mathbf{Z}(n)) \stackrel{\sim}{\longrightarrow} H_{W}^{i}(X, \mathbf{Z}(n)) .
$$

Pour $i>2 d+2$, l'assertion résulte de la dimension cohomologique de $X$; pour $i=2 d+1$, elle résulte de [31, th. 2] et de la suite spectrale de Bloch-Ogus.

COROLlaire 3.10. - Les conjectures de [43, §7] sont vraies pour $X \in B_{\text {tate }}(k)$.

Démonstration. - Rappelons tout d'abord ces conjectures [43, §7] en termes modernes; étant donné une $k$-variété projective lisse $X$ :

1. $H_{\text {ét }}^{i}(X, \mathbf{Z}(n))=0$ pour $i$ grand.

2. $H_{\text {ét }}^{2 n}(X, \mathbf{Z}(n))$ est un groupe abélien de type fini.

3. $H_{\text {ét }}^{i}(X, \mathbf{Z}(n))$ est fini pour $i \neq 2 n, 2 n+2$, nul pour $i \leqslant 0$ lorsque $n>0 .^{3}$

4. $H_{\text {ét }}^{2 d+2}(X, \mathbf{Z}(d))$ est canoniquement isomorphe à $\mathbf{Q} / \mathbf{Z}$, où $d=\operatorname{dim} X$.

5. L'accouplement

$$
H_{\text {ét }}^{i}(X, \mathbf{Z}(n)) \times H_{\text {ét }}^{2 d+2-i}(X, \mathbf{Z}(d-n)) \rightarrow H_{\text {ét }}^{2 d+2}(X, \mathbf{Z}(d)) \stackrel{\sim}{\longrightarrow} \mathbf{Q} / \mathbf{Z}
$$

est "parfait", au sens qu'il définit une dualité parfaite de groupes finis pour $i \neq 2 n$ et une dualité parfaite entre un groupe de type fini et un groupe de cotype fini pour $i=2 n$. En particulier, $\operatorname{rg} H_{\text {ét }}^{2 d}(X, \mathbf{Z}(d))=1$.

6. Les groupes $H_{\text {ét }}^{2 n}(X, \mathbf{Z}(n))$ et $H_{\text {ét }}^{2 d-2 n}(X, \mathbf{Z}(d-n))$ ont le même rang $m(n)$.

7. $m(n)$ est l'ordre du pôle de la fonction $Z(X, t)$ en $t=q^{-n}\left(\zeta(X, s)=Z\left(X, q^{-s}\right)\right)$.

\footnotetext{
${ }^{3}$ Cette conjecture de nullité résulte de l'axiome (1) de [43, §3]; il est pratique de l'insérer ici.
} 
8. $\lim _{t \rightarrow q^{-n}}\left(1-q^{n} t\right)^{m(n)} Z(X, t)= \pm q^{\chi\left(X, \mathcal{O}_{X}, n\right)} \chi(X, \mathbf{Z}(n))$, avec

$$
\begin{aligned}
\chi(X, \mathbf{Z}(n))= & \prod_{i \neq 2 n, 2 n+2}\left|H_{\text {ét }}^{i}(X, \mathbf{Z}(n))\right|^{(-1)^{i}} \\
& \times \frac{\left|H_{\text {ét }}^{2 n}(X, \mathbf{Z}(n))_{\text {tors }}\right|\left|H_{\text {ét }}^{2 n+2}(X, \mathbf{Z}(n))_{\text {cotors }}\right|}{R_{n}(X)}
\end{aligned}
$$

où $R_{n}(X)$ est la valeur absolue du déterminant de l'accouplement

$$
H_{\text {ét }}^{2 n}(X, \mathbf{Z}(n)) / \text { tors } \times H_{\text {ét }}^{2 d-2 n}(X, \mathbf{Z}(d-n)) / \text { tors } \rightarrow H_{\text {ét }}^{2 d}(X, \mathbf{Z}(d)) / \text { tors } \stackrel{\sim}{\longrightarrow} \mathbf{Z}
$$

par rapport à des bases quelconques de $H_{\text {ét }}^{2 n}(X, \mathbf{Z}(n)) /$ tors et de $H_{\text {ét }}^{2 d-2 n}(X, \mathbf{Z}(d-n)) /$ tors, et

$$
\chi\left(X, \mathcal{O}_{X}, n\right)=\sum_{\substack{0 \leqslant i \leqslant n \\ 0 \leqslant j \leqslant d}}(-1)^{i+j}(n-i) h_{i j}, \quad h_{i j}=\operatorname{dim} H^{j}\left(X, \Omega^{i}\right) .
$$

En fait, $X$ étant quelconque, les énoncés 1 et 4 ne sont plus des conjectures, ni le fait que $\operatorname{rg} H_{\text {ét }}^{2 d}(X, \mathbf{Z}(d))=1$ : vu (3.2), le point a) de la proposition 3.3 implique la conjecture 1 de Lichtenbaum pour $i>2 d+2$ et le point b) implique la conjecture 4 et le fait que $H_{\text {ét }}^{2 d}(X, \mathbf{Z}(d))$ est de type fini et de rang 1 . Cela peut d'ailleurs s'obtenir directement.

Il reste à traiter les conjectures $2,3,5,6,7$ et 8 . La conjecture 2 résulte du corollaire $3.8 \mathrm{c}$ ) et e), ainsi que la conjecture 3 pour $i<2 n$; le reste découle du corollaire $3.8 \mathrm{c}$ ) et de la suite exacte (3.2). De même, 5 découle du corollaire 3.8 a) et b), via (3.2), et 6 découle du corollaire 3.8 a) et e). Enfin, 7 découle du corollaire 3.8 e) et du corollaire 2.1 .

Pour 8 , on préfère démontrer la variante de [17, th. 8.1] (voir aussi [53, th. 10.7]), qui lui est équivalente via (3.2) :

$8_{W} \cdot \lim _{t \rightarrow q^{-n}}\left(1-q^{n} t\right)^{m(n)} Z(X, t)= \pm q^{\chi\left(X, \mathcal{O}_{X}, n\right)} \chi(X, \mathbf{Z}(n))$, avec

$$
\chi(X, \mathbf{Z}(n))=\chi\left(H_{W}^{*}(X, \mathbf{Z}(n)), \cdot e\right)=\prod_{i}\left|H_{W}^{i}(X, \mathbf{Z}(n))_{\mathrm{tors}}\right|^{(-1)^{i}} \cdot R_{n}(X)^{-1}
$$

et $\chi\left(X, \mathcal{O}_{X}, n\right)$ comme dans 8 , où $\cdot e$ est vu comme une dérivation de degré 1 du groupe gradué $H_{W}^{*}(X, \mathbf{Z}(n))$ et $R_{n}(X)$ est la valeur absolue du déterminant de l'accouplement (3.11) vu modulo torsion par rapport à des bases quelconques de $H_{W}^{2 n}(X, \mathbf{Z}(n)) /$ tors et de $H_{W}^{2 d-2 n}(X, \mathbf{Z}(d-n)) /$ tors.

On peut procéder comme dans [49, th. 4.3 et cor. 5.5] ( $c f$.[33, cor. 7.10 et th. 9.20] et [17, dém. du th. 8.1]).

Remarque 3.11. - Il est facile de préciser le signe dans la conjecture 8 de Lichtenbaum : on remarque simplement que $\zeta(X, s)$ est à valeurs réelles positives pour $s$ réel assez grand et ne s'annule pas pour $s>d$. Le signe en $s=n$ ne dépend alors que de l'ordre des zéros pour $s>n$ : on trouve qu'il est égal à $(-1)^{\sum_{a>n} m(a)}$.

Corollaire 3.12. - Soit $X \in B_{\text {tate }}(k)$. Alors, pour tout $l$, on a une suite exacte

$$
\begin{aligned}
0 & \rightarrow C H^{n}(X)\{l\} \rightarrow C H^{n}(X) \otimes \mathbf{Z}_{l} \oplus H_{\text {ét }}^{2 n-1}\left(X, \mathbf{Q}_{l} / \mathbf{Z}_{l}(n)\right) \\
& \rightarrow H_{\text {cont }}^{2 n}\left(X, \mathbf{Z}_{l}(n)\right) \rightarrow C H^{n}(X) \otimes \mathbf{Q}_{l} / \mathbf{Z}_{l} \rightarrow H_{\text {ét }}^{2 n}\left(X, \mathbf{Q}_{l} / \mathbf{Z}_{l}(n)\right) .
\end{aligned}
$$

En particulier, on a une suite exacte 


$$
\begin{aligned}
0 & \rightarrow \operatorname{Ker}\left(C H^{n}(X) \otimes \mathbf{Q}_{l} / \mathbf{Z}_{l} \stackrel{\overline{c l}}{\longrightarrow} H_{\text {ét }}^{2 n}\left(X, \mathbf{Q}_{l} / \mathbf{Z}_{l}(n)\right)\right) \\
& \rightarrow \operatorname{Coker}\left(C H^{n}(X) \otimes \mathbf{Z}_{l} \stackrel{c l}{\longrightarrow} H_{\text {cont }}^{2 n}\left(X, \mathbf{Z}_{l}(n)\right)\right) \\
& \rightarrow \operatorname{Coker}\left(C H^{n}(X)\{l\} \stackrel{b l}{\longrightarrow} H_{\text {ét }}^{2 n-1}\left(X, \mathbf{Q}_{l} / \mathbf{Z}_{l}(n)\right)\right) \rightarrow 0
\end{aligned}
$$

où cl est la classe de cycle l-adique, čl est la classe de cycle à coefficients divisibles et bl est [un raffinement du] raffinement de Bloch de la classe de cycle l-adique sur les cycles algébriques de torsion [4].

Démonstration. - Cela résulte immédiatement du diagramme commutatif de suites exactes

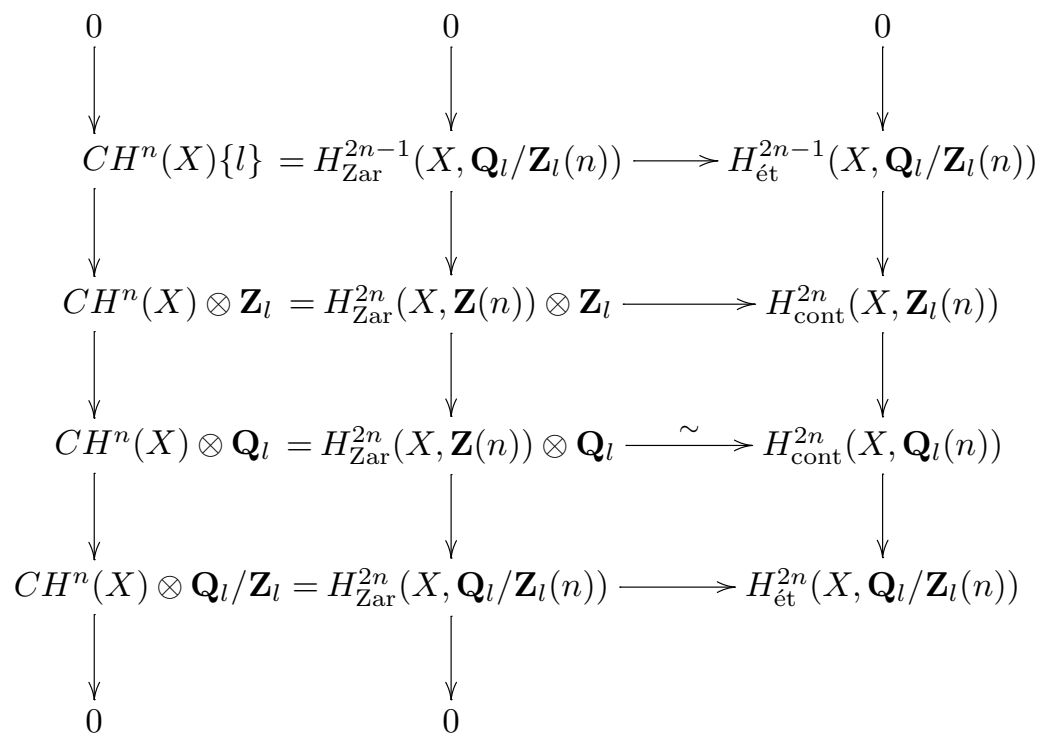

La partie droite de ce diagramme est commutative par construction (les flèches horizontales émanent de la composition de (3.3) et du morphisme $\mathbf{Z}(n) \rightarrow R \alpha_{*} \alpha^{*} \mathbf{Z}(n)$ ). Le 0 supérieur de droite provient de la conjecture de Weil, comme dans [9] (donc est valable pour tout $X$ ). Compte tenu de la proposition 3.1, celui du milieu provient du corollaire $3.8 \mathrm{c}$ ) et e) et l'isomorphisme provient de ce corollaire et du théorème 3.6 (ils utilisent donc le fait que $X \in B_{\text {tate }}(k)$ ). L'égalité (isomorphisme canonique) de la deuxième ligne résulte de Bloch [5,7]; les autres se déduisent de ce qui précède. Le fait que la composition de la deuxième ligne soit la classe de cycle $l$-adique de Jannsen [25, (6.14)] est expliqué dans [35, lemma 1.23]. Enfin, le fait que la flèche horizontale supérieure redonne l'application de Bloch sur $\bar{k}$ peut se voir comme dans Raskind [55, §2].

Corollaire 3.13 (cf. [48, remark 5.6 a)], [53, §3]).-Soit $X \in B_{\text {tate }}(k)$. Pour que l'application cycle l-adique entière $C H^{n}(X) \otimes \mathbf{Z}_{l} \rightarrow H_{\text {cont }}^{2 n}\left(X, \mathbf{Z}_{l}(n)\right)$ soit surjective, il faut et il suffit que

(i) la classe de cycle à coefficients divisibles

$$
C H^{n}(X) \otimes \mathbf{Q}_{l} / \mathbf{Z}_{l} \stackrel{\bar{c} l}{\longrightarrow} H_{\text {ét }}^{2 n}\left(X, \mathbf{Q}_{l} / \mathbf{Z}_{l}(n)\right)
$$

soit injective;

(ii) l'application de Bloch $C H^{n}(X)\{l\} \stackrel{b l}{\longrightarrow} H_{\mathrm{ett}}^{2 n-1}\left(X, \mathbf{Q}_{l} / \mathbf{Z}_{l}(n)\right)$ soit surjective. 


\section{Variétés ouvertes}

LEMME 4.1. - Soit X une $k$-variété projective lisse de dimension d. Alors sous les conditions suivantes

(i) $d \leqslant 1$,

(ii) $n \leqslant 1$,

les groupes $H_{W}^{i}(X, \mathbf{Z}(n))$ sont de type fini pour $i \neq 2 n+1$. Ils sont finis pour $i \neq 2 n, 2 n+1$ ainsi que pour $n>d$, et nuls pour $i \leqslant 0$ si $n>0$.

Démonstration. $-\mathrm{Si} n=0$, l'énoncé résulte de [44, th. 3.2]. Supposons $n=1$ : alors $\mathbf{Z}(1)=\mathbb{G}_{m}[-1]$. La suite exacte (3.2) se retraduit en une suite exacte

$$
\cdots \rightarrow H_{\text {ét }}^{i-1}\left(X, \mathbb{G}_{m}\right) \rightarrow H_{W}^{i}(X, \mathbf{Z}(1)) \rightarrow H_{\text {ét }}^{i-2}\left(X, \mathbb{G}_{m}\right) \otimes \mathbf{Q} \stackrel{\partial}{\rightarrow} H_{\text {ét }}^{i}\left(X, \mathbb{G}_{m}\right) \rightarrow \cdots
$$

Le groupe $H_{\text {ét }}^{i-2}\left(X, \mathbb{G}_{m}\right) \otimes \mathbf{Q}$ est nul pour $i \neq 3$. Le groupe $H_{W}^{i}(X, \mathbf{Z}(1))$ est donc nul pour $i \leqslant 0$, isomorphe à $E^{*}$ pour $i=1$ (où $E$ est le corps des constantes de $X$ ) donc fini, et isomorphe à $\operatorname{Pic}(X)$ pour $i=2$, donc de type fini par le théorème de Néron-Severi. Pour $i>3$, il est isomorphe à un quotient de $H_{\text {ét }}^{i-1}\left(X, \mathbb{G}_{m}\right)$; par la suite exacte de Kummer, ce groupe est luimême isomorphe à $H_{\text {ét }}^{i-1}\left(X,(\mathbf{Q} / \mathbf{Z})^{\prime}(1)\right)$ qui est fini par [9, th. 2]. Enfin, si $X$ est un point, les $H_{\text {ét }}^{i}\left(X, \mathbb{G}_{m}\right)$ sont finis pour tout $i$, donc aussi les $H_{W}^{i}(X, \mathbf{Z}(1))$. Ceci démontre (ii).

Si $X$ est un point ou une courbe, toute la stratégie de démonstration conduisant au corollaire $3.8 \mathrm{~s}$ 'applique : la conjecture de Tate est vraie pour $X$ et l'équivalence rationnelle coïncide avec l'équivalence numérique, donc le théorème 3.6 est vrai pour $X$. On en déduit (i).

Remarque 4.2. - D'après (4.1), la génération finie de $H_{W}^{3}(X, \mathbf{Z}(1))$ implique la finitude de $H_{\text {ét }}^{2}\left(X, \mathbb{G}_{m}\right)=\operatorname{Br}(X)$, elle-même équivalente à la validité de la conjecture de Tate en codimension 1 pour $X$. Cette implication est donc en fait une équivalence par les raisonnements du paragraphe précédent.

LEMME 4.3. - Soit X une $k$-variété lisse de dimension d. Alors sous les conditions suivantes

(i) $d \leqslant 1$,

(ii) $n \leqslant 1$,

les groupes $H_{W}^{i}(X, \mathbf{Z}[1 / p](n))$ sont des $\mathbf{Z}[1 / p]$-modules de type fini pour $i \leqslant n$ ou $i>2 n+1$. Ils sont finis pour $i \notin[n, 2 n+1]$ ainsi que pour $n>d$, et nuls pour $i \leqslant 0$ si $n>0$.

Démonstration. - En partant du lemme 4.1, on utilise la même méthode de dévissage que celle de [36, dém. du th. 1] : c'est loisible car les groupes $H_{W}^{i}(X, \mathbf{Z}[1 / p](n))$ satisfont à un théorème de pureté, ce qui se réduit à la pureté de la cohomologie étale [35, cor. 1.19] via l'isomorphisme (3.6) (c'est pour avoir cette pureté qu'il est nécessaire d'inverser $p$ ). Le seul point à vérifier est qu'on ne "perd" pas de génération finie quand on utilise le théorème de de Jong [28].

Soit donc $\tilde{U} \rightarrow U$ un revêtement fini de variétés lisses, et supposons que $H_{W}^{i}(\tilde{U}, \mathbf{Z}[1 / p](n))$ soit un $\mathbf{Z}[1 / p]$-module de type fini. Par un argument de transfert,

$$
\operatorname{Ker}\left(H_{W}^{i}(U, \mathbf{Z}[1 / p](n)) \rightarrow H_{W}^{i}(\tilde{U}, \mathbf{Z}[1 / p](n))\right)
$$

est d'exposant fini, disons $m$. Mais $H_{\text {ét }}^{i-1}\left(U, \mu_{m}^{\otimes n}\right)=H_{W}^{i-1}\left(U, \mu_{m}^{\otimes n}\right)$ est fini par [13, Th. finitude], ce qui montre que ce noyau est fini, d'où la génération finie de $H_{W}^{i}(U, \mathbf{Z}[1 / p](n))$.

La proposition suivante raffine le corollaire 2.5 :

Proposition 4.4. - Soit $U$ un ouvert non vide de $X$, où $X \in B_{\text {tate }}(k)$, et soit

$$
d=\operatorname{dim} X=\operatorname{dim} U \text {. }
$$


Alors, dans les deux cas suivants:

(i) $d \leqslant 2$,

(ii) $n \leqslant 2$,

les conclusions du lemme 4.3 sont vraies pour $U$.

Démonstration. - Même méthode que ci-dessus, en utilisant le corollaire 3.8 c) et le lemme 4.3. Plus précisément :

Notons $Z=X-U$ (structure réduite) et stratifions $Z$ par sa chaîne canonique de lieux singuliers $\left(Z_{0}=Z, Z_{r+1}=\left(Z_{r}\right)_{\text {sing }}\right)$. Il suffit de voir que, pour tout $r \geqslant 0$, le groupe

$$
H_{W}^{j}\left(Z_{r}-Z_{r+1}, \mathbf{Z}[1 / p]\left(n-c_{r}\right)\right)
$$

vérifie les hypothèses du lemme 4.3, où $c_{r}$ est la codimension de $Z_{r}$ dans $X$.

a) $\mathrm{Si} d \leqslant 2$, les $Z_{r}-Z_{r-1}$ sont soit des points soit des courbes, et on est dans le cas (i) du lemme 4.3.

b) Si $n \leqslant 2$, on a $n-c_{r} \leqslant 1$ pour tout $r$, et on est dans le cas (ii) du lemme 4.3.

THÉORÈME 4.5. - Soient $X \in B_{\text {tate }}(k)$ et $U$ un ouvert de $X$. Alors les groupes

$$
H^{0}\left(U, \mathcal{K}_{2}\right), H^{1}\left(U, \mathcal{K}_{2}\right), H^{2}\left(U, \mathcal{K}_{2}\right)=C H^{2}(U)
$$

sont de type fini. Le groupe $K_{3}(K)_{\text {ind }}$ est égal à $K_{3}(k)$, où $K$ est le corps des fonctions de $X$ (ou de $U$ ).

Démonstration. - Si l'on acceptait d'inverser $p$, ce résultat se déduirait directement de la proposition 4.4 via [32, th. 1.1 et 1.6]; pour obtenir un résultat exact, on est obligé de refaire la démonstration (pureté) avec la cohomologie de Zariski motivique plutôt qu'avec la cohomologie de Lichtenbaum motivique.

Traitons d'abord le cas de $X$ : d'après [32, th. 1.6], les groupes cités ne sont autres que $H^{i}(X, \mathbf{Z}(2))$ pour $i$ respectivement égal à $2,3,4$ et 1 . D'autre part, d'après loc. cit., th. 1.1, l'homomorphisme $H^{i}(X, \mathbf{Z}(2)) \rightarrow H_{\text {ét }}^{i}(X, \mathbf{Z}(2))$ est bijectif pour $i \leqslant 3$ et injectif pour $i=4$. L'énoncé pour les $H^{i}\left(X, \mathcal{K}_{2}\right)$ résulte donc encore du corollaire $\left.3.8 \mathrm{c}\right)$. De plus, on obtient que $K_{3}(K)_{\text {ind }}$ est de type fini. Mais, d'après Merkurjev et Suslin [46], l'homomorphisme

$$
K_{3}(k) \rightarrow K_{3}(K)_{\text {ind }}
$$

est injectif à conoyau divisible; il est donc bijectif (cf. [29]).

(Dans [32], nous travaillions avec le complexe $\Gamma(2)$ de Lichtenbaum : les raisonnements sont identiques en remplaçant $\Gamma(2)$ par $\mathbf{Z}(2)$ et en utilisant les résultats de Merkurjev et Suslin [45, 46].)

Dans le cas général, on raisonne comme dans la démonstration de la proposition 4.4, en utilisant le théorème de pureté pour la cohomologie motivique et le fait que les groupes $H_{\text {Zar }}^{i}(X, \mathbf{Z}(m))$ sont de type fini pour $m \leqslant 1$ et toute $k$-variété lisse $X:$ pour $m=0$ c'est évident et pour $m=1$ cela se réduit à la génération finie de $\Gamma\left(X, \mathbb{G}_{m}\right)(i=1)$ et de $\operatorname{Pic}(X)$ $(i=2){ }^{4}$

\footnotetext{
${ }^{4}$ On peut réduire la preuve de la génération finie de $\operatorname{Pic}(X)$ au cas projectif en passant par le théorème de de Jong, par le même raisonnement que dans la démonstration du lemme 4.3.
} 
THÉORÈmE 4.6. - Soient $X \in B_{\text {tate }}(k)$ de dimension $\leqslant 2$ et $K=k(X)$. Alors on a des isomorphismes canoniques

$$
\left(K_{n}^{M}(K) \oplus \bigoplus_{0 \leqslant i \leqslant n-1} H^{2 i-n-1}\left(K,(\mathbf{Q} / \mathbf{Z})^{\prime}(i)\right)\right) \otimes \mathbf{Z}_{(2)} \stackrel{\sim}{\longrightarrow} K_{n}(K) \otimes \mathbf{Z}_{(2)}
$$

où

$$
(\mathbf{Q} / \mathbf{Z})^{\prime}(i)=\underset{(m, \operatorname{car} k)=1}{\lim _{(}} \mu_{m}^{\otimes i} .
$$

De plus, $K_{n}^{M}(K)$ est de torsion pour $n \geqslant 3$ et nul pour $n \geqslant 4$.

Démonstration. - Supposons d'abord car $k=2$. Alors le second facteur du membre de gauche est nul. L'isomorphisme $K_{n}^{M}(K) \otimes \mathbf{Z}_{(2)} \stackrel{\sim}{\longrightarrow} K_{n}(K) \otimes \mathbf{Z}_{(2)}$ résulte de [18].

Supposons maintenant car $k \neq 2$. Pour construire le morphisme, on part des isomorphismes de $[34$, th. 1] :

$$
\bigoplus_{0 \leqslant i \leqslant n+1} H_{\text {ét }}^{2 i-n-1}\left(K, \mu_{2^{\nu}}^{\otimes i}\right) \stackrel{\sim}{\longrightarrow} K_{n+1}\left(K, \mathbf{Z} / 2^{\nu}\right) .
$$

En prenant la limite inductive sur $\nu$, on obtient des isomorphismes

$$
\bigoplus_{0 \leqslant i \leqslant n+1} H_{\text {ét }}^{2 i-n-1}\left(K, \mathbf{Q}_{2} / \mathbf{Z}_{2}(i)\right) \stackrel{\sim}{\longrightarrow} K_{n+1}\left(K, \mathbf{Q}_{2} / \mathbf{Z}_{2}\right) .
$$

On obtient l'homomorphisme de l'énoncé en composant avec le Bockstein

$$
K_{n+1}\left(K, \mathbf{Q}_{2} / \mathbf{Z}_{2}\right) \rightarrow K_{n}(K) \otimes \mathbf{Z}_{(2)}
$$

et en négligeant les facteurs $i=n, n+1$.

Pour montrer que cet homomorphisme est un isomorphisme, on raisonne comme dans [34] en utilisant la suite spectrale (convergente) de Bloch-Lichtenbaum [8]

$$
E_{2}^{p, q}=H^{p-q}(K, \mathbf{Z}(-q)) \Rightarrow K_{-p-q}(K) .
$$

Tout d'abord, le corollaire 4.4 montre que le groupe $H_{\mathrm{Zar}}^{i}(K, \mathbf{Z}(n))$ est de torsion pour $i<n$. Il en résulte que le Bockstein $H_{\mathrm{Zar}}^{i-1}\left(K,(\mathbf{Q} / \mathbf{Z})^{\prime}(n)\right) \rightarrow H_{\mathrm{Zar}}^{i}(K, \mathbf{Z}[1 / p](n))$ est un isomorphisme pour $i<n$. D'autre part, la conjecture de Milnor [64] et [19] montrent que les homomorphismes $H_{\mathrm{Zar}}^{i-1}\left(K, \mathbf{Q}_{2} / \mathbf{Z}_{2}(n)\right) \rightarrow H_{\text {ét }}^{i-1}\left(K, \mathbf{Q}_{2} / \mathbf{Z}_{2}(n)\right)$ sont des isomorphismes pour $i \leqslant n$. On en déduit des isomorphismes

$$
H_{\text {ét }}^{i-1}\left(K, \mathbf{Q}_{2} / \mathbf{Z}_{2}(n)\right) \stackrel{\sim}{\longrightarrow} H_{\text {Zar }}^{i}(K, \mathbf{Z}(n)) \otimes \mathbf{Z}_{(2)}, \quad i<n .
$$

En utilisant la compatibilité de (4.2) aux produits et aux transferts [14,42] et les isomorphismes (4.3), et en examinant la construction des homomorphismes du théorème 4.4, on démontre comme dans $[34, \S 3]$ que ces derniers détruisent successivement les différentielles de la suite spectrale (4.2) (localisée en 2) et scindent la filtration donnée par celle-ci sur l'aboutissement. Ceci conclut la démonstration, et donne de plus que la suite spectrale (4.2) dégénère canoniquement après localisation en 2.

Corollaire 4.7. - L'algèbre $K_{*}(K) \otimes \mathbf{Z}_{(2)}$ est engendrée par les unités et la $K$-théorie $d u$ sous-corps des constantes, à transfert près. 
Démonstration. - Cela résulte de la construction dans [34] des flèches donnant naissance à l'isomorphisme du théorème 4.6. Plus précisément, l'homomorphisme composé

$$
\begin{aligned}
& \bigoplus_{\left[k^{\prime}: k\right]<\infty} K_{2 i-n-1}^{M}\left(k^{\prime} K\right) \otimes H^{0}\left(k^{\prime}, \mathbf{Q}_{2} / \mathbf{Z}_{2}(n+1-i)\right) \\
& \quad \rightarrow \bigoplus_{\left[k^{\prime}: k\right]<\infty} H^{2 i-n-1}\left(k^{\prime} K, \mathbf{Q}_{2} / \mathbf{Z}_{2}(i)\right) \stackrel{\text { Cor }}{\longrightarrow} H^{2 i-n-1}\left(k^{\prime} K, \mathbf{Q}_{2} / \mathbf{Z}_{2}(i)\right)
\end{aligned}
$$

est surjectif pour tout $0 \leqslant i \leqslant n-1$ d'après la conjecture de Milnor et [30, th. 1]. D'autre part, on a d'après Quillen des isomorphismes $K_{2 n-2 i+1}^{M}\left(k^{\prime}\right) \otimes \mathbf{Z}_{(2)} \stackrel{\sim}{\longrightarrow} H^{0}\left(k^{\prime}, \mathbf{Q}_{2} / \mathbf{Z}_{2}(n+1-i)\right)$. Enfin, la composition de la surjection induite

$$
\bigoplus_{\left[k^{\prime}: k\right]<\infty} K_{2 i-n-1}^{M}\left(k^{\prime} K\right) \otimes K_{2 n-2 i+1}\left(k^{\prime}\right) \otimes \mathbf{Z}_{(2)} \rightarrow H^{2 i-n-1}\left(K, \mathbf{Q}_{2} / \mathbf{Z}_{2}(i)\right)
$$

avec la flèche du théorème 4.6 n'est autre par construction ( $c f$. [34]) que celle donnée par le produit en $K$-théorie. Le corollaire en résulte.

Remarque 4.8. - En passant à la limite sur les extensions finies de $k$, on déduit du corollaire 4.7 que la conjecture de Suslin [60, conj. 4.1 et note] est vraie après localisation en 2 pour le corps $L=\bar{k} K:$ l'algèbre $K_{*}(L) \otimes \mathbf{Z}_{(2)}$ est engendrée par $K_{1}(L) \otimes \mathbf{Z}_{(2)}$ et $K_{*}(\bar{k}) \otimes \mathbf{Z}_{(2)}$.

Remarque 4.9. - Soit $U$ un ouvert de $X$, où $X \in B_{\text {tate }}(k)$ et $\operatorname{dim} X \leqslant 2$. On peut montrer que, pour tout $n \in \mathbf{Z}$

(i) $\zeta(U, s)=\left(1-q^{n-s}\right)^{a_{n}} \varphi(s)$, avec $a_{n}=\sum(-1)^{r+1} \operatorname{rg} H_{r}^{c, \text { ét }}(U, \mathbf{Z}(n))$ où

(ii) $\varphi(n)=\prod_{r=0}^{2 d} \operatorname{ind}\left(\bar{\partial}_{r}\right)^{(-1)^{r+1}}$ à une puissance de $q$ près

a) $H_{r}^{c, \text { ét }}(U, \mathbf{Z}(n))=H_{\text {ét }}^{2 d-r}(U, \mathbf{Z}(d-n))$

b) $\bar{\partial}_{r}: H_{r+2}^{c \text { ét }}(U, \mathbf{Z}(n)) \otimes(\mathbf{Q} / \mathbf{Z})^{\prime} \rightarrow H_{r}^{c, \text { ét }}(U, \mathbf{Z}(n))_{\text {tors }}[1 / p]$ est un certain homomorphisme induit par l'homomorphisme $\partial$ de (3.2), dont le noyau et le conoyau sont finis

c) $\operatorname{ind}\left(\bar{\partial}_{r}\right)=\left|\operatorname{Ker} \bar{\partial}_{r}\right| / \mid$ Coker $\bar{\partial}_{r} \mid$.

Voir [33, th. 9.16].

Remarque 4.10. - Si l'on essaye d'étendre les résultats du théorème 4.5 aux poids supérieurs, on se heurte d'abord à la conjecture de Bloch-Kato (isomorphisme de la $K$-théorie de Milnor modulo $m$ avec la cohomologie galoisienne). Pour les besoins de la discussion, supposons celleci connue. Alors le résultat principal de Geisser et Levine [19] implique que le morphisme canonique

$$
\mathbf{Z}(n) \rightarrow \tau_{\leqslant n+1} R \alpha_{*} \alpha^{*} \mathbf{Z}(n)
$$

est un quasi-isomorphisme dans $D^{-}\left((S m / k)_{\text {Zar }}\right)$. Concrètement, on en déduit que, pour toute $k$-variété lisse $X$, l'homomorphisme

$$
H_{\text {Zar }}^{i}(X, \mathbf{Z}(n)) \rightarrow H_{\text {ét }}^{i}(X, \mathbf{Z}(n))
$$

est un isomorphisme pour $i \leqslant n+1$ et est injectif pour $i=n+2$. Si $X$ est par exemple un produit de courbes elliptiques, cela implique via le corollaire $3.8 \mathrm{c}$ ) que $H_{\mathrm{Zar}}^{i}(X, \mathbf{Z}(n))$ est de type fini pour $i \leqslant n+2$.

Pour $n \leqslant 2$, ceci couvre toute la cohomologie motivique de $X$ : c'est le théorème 4.5. Examinons le cas $n=3$. On obtient une suite exacte : 


$$
\begin{aligned}
0 & \rightarrow H_{\text {Zar }}^{5}(X, \mathbf{Z}(3)) \rightarrow H_{\text {ét }}^{5}(X, \mathbf{Z}(3)) \rightarrow H_{\text {Zar }}^{0}\left(X, R^{5} \alpha_{*} \mathbf{Z}(3)\right) \\
& \rightarrow C H^{3}(X) \rightarrow H_{\text {ét }}^{6}(X, \mathbf{Z}(3)) .
\end{aligned}
$$

Ceci montre que sous la conjecture de Bloch-Kato et pour $X \in B_{\text {tate }}(k), C H^{3}(X)$ est de type fini si et seulement si le groupe de cohomologie non ramifiée

$$
H_{\text {Zar }}^{0}\left(X, R^{5} \alpha_{*} \mathbf{Z}(3)\right) \simeq H_{\text {Zar }}^{0}\left(X, \mathcal{H}_{\text {ét }}^{4}\left((\mathbf{Q} / \mathbf{Z})^{\prime}(3)\right)\right)
$$

est [de type] fini.

Pour rendre la discussion ci-dessus inconditionnelle, on peut localiser en $l=2$ et utiliser le théorème de Voevodsky [64]. On peut aussi localiser en $l=p$ et utiliser le théorème de GeisserLevine [18].

On peut aller un peu plus loin en interprétant $H_{\mathrm{Zar}}^{0}\left(X, R^{5} \alpha_{*} \mathbf{Z}(3)\right)$ comme un groupe de Chow à coefficients à la Rost [56], via la "conjecture de Gersten" [10]. Cette interprétation montre immédiatement que les correspondances de Chow opèrent sur les $H_{\mathrm{Zar}}^{p}\left(X, R^{q} \alpha_{*} \mathbf{Z}(n)\right)$. En utilisant la formule du fibré projectif pour ces groupes [20], on en déduit que la notation $H_{\text {Zar }}^{p}\left(M, R^{q} \alpha_{*} \mathbf{Z}(n)\right)$ a un sens pour tout motif de Chow $M$, avec

$$
H_{\mathrm{Zar}}^{p}\left(M(1), R^{q} \alpha_{*} \mathbf{Z}(n)\right)=H_{\mathrm{Zar}}^{p-1}\left(M, R^{q-1} \alpha_{*} \mathbf{Z}(n-1)\right) .
$$

D'autre part, Frobenius opère sur $H_{\mathrm{Zar}}^{p}\left(M, R^{q} \alpha_{*} \mathbf{Z}(n)\right)$ par multiplication par $q^{n}$ : se ramener au cas d'une variété projective lisse et passer par l'interprétation à la Rost, en raisonnant comme dans [57, prop. 2]. Si $M$ est un facteur direct simple de $h(X)=\bar{h}(X)$, on en déduit que $P_{M}\left(q^{n}\right) H_{\text {Zar }}^{p}\left(M, R^{q} \alpha_{*} \mathbf{Z}(n)\right)=0$, donc que $H_{\text {Zar }}^{p}\left(M, R^{q} \alpha_{*} \mathbf{Z}(n)\right)$ est d'exposant fini si $M \neq L^{n}$. Pour $p=0$ et $n>0$, on a $H_{\mathrm{Zar}}^{p}\left(L^{n}, R^{q} \alpha_{*} \mathbf{Z}(n)\right)=0$; on en conclut, inconditionnellement, que si $X \in B_{\text {tate }}(k), H_{\mathrm{Zar}}^{0}\left(X, R^{5} \alpha_{*} \mathbf{Z}(3)\right)$ est d'exposant fini. Je ne sais pas faire mieux.

Il serait amusant de donner un exemple où la 2-torsion de $H_{\mathrm{Zar}}^{0}\left(X, \mathcal{H}_{\text {ét }}^{4}\left((\mathbf{Q} / \mathbf{Z})^{\prime}(3)\right)\right.$ est infinie.

\section{Prospective}

Rappelons le résultat principal de [35] :

THÉORÈME 5.1 ([35, th. 3.4]). - Les trois conjectures suivantes sont équivalentes:

(i) Le théorème 1.10 (avec $X^{\prime}=\operatorname{Spec} k$ ) et le corollaire 2.1 sont vrais pour toute $k$-variété projective lisse $X$.

(ii) Pour tout $n>0$, le morphisme $\Phi_{n}$ du lemme 3.5 (relatif à un nombre premier $l \neq p$ donné) est un quasi-isomorphisme.

(iii) Pour tout $n>0$, le complexe $\mathbf{Z}_{l}(n)_{\text {ét }}^{c}$ (relatif à un nombre premier $l \neq p$ donné) est malléable (voir [35, déf. 2.16] pour la définition de malléable).

Les méthodes précédentes conduisent en fait à d'autres formulations de ces conjectures :

THÉORÈME 5.2. - Les conjectures du théorème 5.1 sont encore équivalentes aux suivantes:

(iv) Pour tout $n>0$, le morphisme (3.4) (relatif à un nombre premier $l \neq p$ donné) est un quasi-isomorphisme dans $\bar{D}\left(\mathcal{T}_{\mathbf{Z}}\right)$ (cf. mise en garde 3.2.1).

(iv bis) Pour tout $n>0$, le morphisme (3.5) ou (3.8) (relatif à un nombre premier l donné) est un isomorphisme pour toute $k$-variété projective lisse $X$.

(iv ter) Pour tout $n>0$, tout nombre premier l et toute $k$-variété projective lisse $X$, les morphismes (3.5) et (3.8) sont des isomorphismes. 
(v) Pour toute $k$-variété projective lisse $X$, les groupes $H_{W}^{i}(X, \mathbf{Z}(n))$ sont de type fini.

(v bis) Pour toute $k$-variété lisse $X$, les groupes $H_{W}^{i}(X, \mathbf{Z}[1 / p](n))$ sont des $\mathbf{Z}[1 / p]$ modules de type fini.

(v ter) Pour toute $k$-variété projective lisse $X$, les groupes $H_{W}^{i}\left(X, \mathbf{Z}_{(l)}(n)\right)$ sont des $\mathbf{Z}_{(l)}$-modules de type fini, où l est un nombre premier donné.

(vi) Pour toute $k$-variété projective lisse $X$, la forme forte de la conjecture de Tate (concernant $\zeta(X, s)$ ) est vraie et le motif de Chow $h(X)$ est de dimension finie au sens de Kimura. ${ }^{5}$

(vi bis) Pour toute $k$-variété projective lisse $X$, la forme forte de la conjecture de Tate est vraie et l'algèbre des correspondances de Chow $A=C H^{\operatorname{dim} X}(X \times X) \otimes \mathbf{Q}$ est semi-primaire : son radical de Jacobson $R$ est nilpotent et $A / R$ est semi-simple.

(vi ter) La conjecture de Tate (cohomologique, relative à un nombre premier l donné) est vraie pour les $k$-variétés abéliennes et la catégorie rigide $\mathcal{A}$ des $k$-motifs de Chow à coefficients rationnels est engendrée par les motifs de variétés abéliennes et les motifs d'Artin.

(vi quater) La forme forte de la conjecture de Tate est vraie pour toutes les variétés projectives lisses et il n'existe pas de "motif de Chow fantôme" : si $M \in \mathcal{A}$ est tel que $\bar{M}=0$ dans $\overline{\mathcal{A}}$, alors $M=0$.

Démonstration. - (ii) $\Leftrightarrow$ (iv) : cela résulte du lemme 3.5, puisque le foncteur $\bar{R} \gamma_{*}$ est conservatif.

(i) $\Rightarrow$ (iv ter) : on procède comme dans la preuve du théorème 3.6.

(iv ter) $\Rightarrow(\mathrm{v})$ : on procède comme dans la preuve du corollaire 3.8 .

$(\mathrm{v}) \Rightarrow$ (v bis) : on procède comme dans la démonstration du lemme 4.3.

(v bis) $\Rightarrow$ (v ter) pour $l \neq p:$ c'est évident.

(v) $\Rightarrow$ (v ter) : c'est évident.

(v ter) $\Rightarrow$ (iv bis) : notons $K(n)$ le cône de (3.4). L'hypothèse implique que, pour toute $k$ variété projective lisse $X$, les groupes $H_{W}^{i}(X, K(n))$ sont des $\mathbf{Z}_{l}$-modules de type fini. D'autre part, on sait que (3.4) $\otimes{ }^{L} \mathbf{Z} / l$ est un quasi-isomorphisme ([19, th. 1.5] ou [18] selon que $l \neq p$ ou que $l=p)$. Par conséquent, les $H_{W}^{i}(X, K(n))$ sont uniquement divisibles, donc nuls.

(iv bis) $\Rightarrow$ (iv) : on procède comme dans $[35$, lemme 3.8$]$ : réduction à $(3.4) \otimes \mathbf{Q}$, puis pureté et théorème de de Jong.

(i) $\Rightarrow$ (vi ter) : la première partie de (vi ter) résulte de (i) d'après [63]. La deuxième partie est une conséquence classique de la conjecture de Tate forte pour toutes les variétés (qui résulte de (i)) pour $\overline{\mathcal{A}}, c f$. [50, rem. 2.7]; mais (i) implique que $\mathcal{A} \rightarrow \overline{\mathcal{A}}$ est une équivalence de catégories.

(vi ter) $\Rightarrow$ (vi) : c'est clair, puisque le motif d'une variété abélienne est de dimension finie au sens de Kimura [39, ex. 9.1], ainsi que tout motif d'Artin, que la forme forte de la conjecture de Tate résulte de la forme cohomologique et de la semi-simplicité de l'action de Frobenius sur la cohomologie [63], et que cette dernière est vraie pour les variétés abéliennes [65].

(vi) $\Rightarrow$ (vi bis) : cela résulte de [2, prop. 9.1.14] et du théorème de Jannsen [26].

(vi bis) $\Rightarrow$ (i) : on procède comme dans la démonstration du théorème 1.10 et du corollaire 2.1.

(i) $\Rightarrow$ (vi quater) est clair. (vi quater) $\Rightarrow$ (vi ter) : notons $\mathcal{A}_{\text {ab }}$ la sous-catégorie rigide de $\mathcal{A}$ engendrée par les motifs de variétés abéliennes et les motifs d'Artin, et $\overline{\mathcal{A}}_{\mathrm{ab}}$ son image dans $\overline{\mathcal{A}}$. La forme forte de la conjecture de Tate implique que $\overline{\mathcal{A}}_{\mathrm{ab}}=\overline{\mathcal{A}}$ (voir ci-dessus). Soit $X$ une variété projective lisse : il existe des isomorphismes inverses $\bar{f}: \bar{h}(X) \stackrel{\sim}{\longrightarrow} \bar{N}, \bar{g}: N \stackrel{\sim}{\longrightarrow} \bar{h}(X)$, avec $N \in \mathcal{A}_{\mathrm{ab}}$. Relevons $\bar{f}$ et $\bar{g}$ en des morphismes $f, g$ de $\mathcal{A}$. Comme $N$ est de dimension

\footnotetext{
${ }^{5}$ Kimura conjecture le second énoncé sur tout corps de base : cela résulterait des conjectures standard et de l'existence d'une décomposition de Chow-Künneth à la Murre, $c f$. [2, ex. 9.2.4]
} 
finie au sens de Kimura, l'algèbre $\mathcal{A}(N, N)$ est semi-primaire, donc $1_{N}-f g$ est nilpotent et quitte à modifier $f$ ou $g$ on peut supposer que $f g=1_{N}$. On a alors $h(X) \simeq N \oplus M$, avec $M$ fantôme.

La formulation de la conjecture des théorèmes 5.1 et 5.2 qui nous semble la plus prometteuse est (vi ter), mais nous nous garderons bien de faire une conjecture sur la démonstration d'une conjecture!

\section{RÉFÉRENCES}

[1] ANDRÉ Y., Cycles de Tate et cycles motivés sur les variétés abéliennes en caractéristique $\mathrm{p}>0$, prépublication, 2003.

[2] ANDRÉ Y., KAHN B., Nilpotence, radicaux et structures monoïdales (avec un appendice de Peter O'Sullivan), Rend. Sem. Math. Univ. Padova 108 (2002) 107-291.

[3] BeILinson A.A., Height pairings between algebraic cycles, in: Lect. Notes in Math., vol. 1289, Springer, 1987, pp. 1-26.

[4] BLOCH S., Torsion algebraic cycles and a theorem of Rơtman, Compositio Math. 39 (1979) 107-127.

[5] BLOCH S., Algebraic cycles and higher $K$-theory, Adv. Math. 61 (1986) 267-304.

[6] BLOCH S., Algebraic cycles and the Beilinson conjectures, in: The Lefschetz Centennial Conference (Mexico City, 1984), in: Contemp. Math., vol. 58(I), Amer. Math. Society, Providence, RI, 1986, pp. 65-79.

[7] BLOCH S., The moving lemma for higher Chow groups, J. Alg. Geom. 3 (1994) 537-568.

[8] BLOCH S., LichtenBAUM S., A spectral sequence for motivic cohomology, prépublication, 1996.

[9] Colliot-ThélÈne J.-L., SANSUC J.-J., Soulé C., Torsion dans le groupe de Chow de codimension 2, Duke Math. J. 50 (1983) 763-801.

[10] Colliot-ThélÈne J.-L., Hoobler R.T., Kahn B., The Bloch-Ogus-Gabber theorem, in: Fields Institute for Research in Mathematical Sciences Communications Series, vol. 16, Amer. Math. Society, Providence, RI, 1997, pp. 31-94.

[11] Deligne P., La conjecture de Weil, I, Publ. Math. IHÉS 43 (1974) 5-77.

[12] Deligne P., KATZ N., Groupes de Monodromie en géométrie algébrique (SGA 7) II, in: Lect. Notes in Math., vol. 340, Springer, 1973.

[13] Deligne P., et al., Cohomologie étale (SGA 4 1/2), in: Lect. Notes in Math., vol. 569, Springer, 1977.

[14] Friedlander E., Suslin A., The spectral sequence relating algebraic $K$-theory and motivic cohomology, Ann. Sci. Éc. Norm. Sup. 35 (2002) 773-875.

[15] Gabber O., Sur la torsion dans la cohomologie $l$-adique d'une variété, C. R. Acad. Sci. Paris 297 (1983) 179-182.

[16] GEISSER T., Tate's conjecture, algebraic cycles and rational $K$-theory in characteristic $p, K$-Theory 13 (1998) 109-122.

[17] GeISSER T., Weil-étale motivic cohomology, prépublication, 2002, http://www.math.uiuc.edu/Ktheory\#565.

[18] Geisser T., Levine M., The $K$-theory of fields in characteristic $p$, Invent. Math. 139 (2000) 459493.

[19] Geisser T., Levine M., The Bloch-Kato conjecture and a theorem of Suslin-Voevodsky, J. Reine Angew. Math. 530 (2001) 55-103.

[20] Gillet H., Riemann-Roch theorems for higher algebraic $K$-theory, Adv. Math. 40 (1981) 203-289.

[21] Gillet H., Gersten's conjecture for the $K$-theory with torsion coefficients of a discrete valuation ring, J. Algebra 103 (1986) 377-380.

[22] GRAYSON D., Finite generation of the $K$-groups of a curve over a finite field (after D. Quillen), in: Lect. Notes in Math., vol. 966, Springer, 1982, pp. 69-90.

[23] Harder G., Die Kohomologie $S$-arithmetischer Gruppen über Funktionenkörpern, Invent. Math. 42 (1977) 135-175.

[24] IzHboldin O., On $p$-torsion in $K_{*}^{M}$ for fields of characteristic $p$, in: Algebraic K-Theory, in: Soviet Math., vol. 4, Amer. Math. Society, Providence, RI, 1991, pp. 129-144. 
[25] Jannsen U., Continuous étale cohomology, Math. Ann. 280 (1988) 207-245.

[26] JANNSEN U., Motives, numerical equivalence and semi-simplicity, Invent. Math. 107 (1992) 447-452.

[27] DE JEU R., On $K_{4}^{(3)}$ of curves over number fields, Invent. Math. 125 (1996) 523-556.

[28] DE Jong A.J., Smoothness, semi-stability and alterations, Publ. Math. IHÉS 83 (1996) 51-93.

[29] Kahn B., $K_{3}$ d'un schéma régulier, C. R. Acad. Sci. Paris 315 (1992) 433-436.

[30] Kahn B., Deux théorèmes de comparaison en cohomologie étale; applications, Duke Math. J. 69 (1993) 137-165.

[31] KAHN B., Résultats de "pureté" pour les variétés lisses sur un corps fini, in: Algebraic K-Theory and Algebraic Topology, in: NATO ASI Series, Ser. C, vol. 407, Kluwer, 1993, pp. 57-62.

[32] Kahn B., Applications of weight-two motivic cohomology, Doc. Math. 1 (1996) 395-416.

[33] KAHN B., A sheaf-theoretic reformulation of the Tate conjecture, prépublication de l'Institut de Mathématiques de Jussieu no 150, 1998 math.AG/9801017.

[34] KAHN B., $K$-theory of semi-local rings with finite coefficients and étale cohomology, $K$-theory 25 (2002) 99-139.

[35] KAHN B., The Geisser-Levine method revisited and algebraic cycles over a finite field, Math. Ann. 324 (2002) 581-617.

[36] KaHn B., Some finiteness results for étale cohomology, J. Number Theory 99 (2002) 57-73.

[37] Katsura T., Shioda T., On Fermat varieties, Tôhoku Math. J. 31 (1979) 97-115.

[38] KatZ N., Messing W., Some consequences of the Riemann hypothesis for varieties over finite fields, Invent. Math. 23 (1974) 73-77.

[39] Kimura S.I., Chow motives can be finite-dimensional, in some sense, J. Alg. Geom., à paraître.

[40] Kratzer C., $\lambda$-structure en $K$-théorie algébrique, Comment. Math. Helv. 55 (1970) 233-254.

[41] Lenstra H.W., ZARHIN Y.G., The Tate conjecture for almost ordinary abelian varieties over finite fields, in: Advances in Number Theory (Kingston, ON, 1991), Oxford Univ. Press, 1993, pp. 179_ 194.

[42] LEVINE M., K-theory and motivic cohomology of schemes, I, prépublication, 2001.

[43] Lichtenbaum S., Values of zeta functions at non-negative integers, in: Lect. Notes in Math., vol. 1068, Springer, 1984, pp. 127-138.

[44] Lichtenbaum S., The Weil-étale topology, prépublication, 2001.

[45] Merkurjev A., Suslin A., $K$-cohomologie des variétés de Severi-Brauer et homomorphisme de reste normique, Izv. Akad. Nauk SSSR 46 (1982) 1011-1046, (en russe), Trad. anglaise: Math. USSR Izvestiya 21 (1983) 307-340.

[46] MerkuRJev A.S., SuSLin A.A., Le groupe $K_{3}$ pour un corps, Izv. Akad. Nauk SSSR 54 (1990) 339-356, (en russe), Trad. anglaise: Math. USSR Izv. 36 (1990) 541-565.

[47] Milne J.S., Etale Cohomology, Princeton Univ. Press, Princeton, 1980.

[48] MiLne J.S., Values of zeta functions of varieties over finite fields, Amer. J. Math. 108 (1986) 297-360.

[49] Milne J.S., Motivic cohomology and values of the zeta function, Compositio Math. 68 (1988) 59102.

[50] Milne J.S., Motives over finite fields, in: Motives (Seattle, WA, 1991), in: Proc. Sympos. Pure Math., vol. 55(1), Amer. Math. Society, Providence, RI, 1994, pp. 401-459.

[51] MiLne J.S., Lefschetz motives and the Tate conjecture, Compositio Math. 117 (1999) 47-81.

[52] MiLne J.S., The Tate conjecture for certain abelian varieties over finite fields, Acta Arith. 100 (2001) $135-166$.

[53] Milne J.S., RAMACHANDRAN N., Integral motives and special values of zeta functions, prépublication, 2002 (version préliminaire), math.NT/0204065.

[54] QuilLEN D., On the cohomology and the $K$-theory of the general linear group over a finite field, Ann. of Math. 96 (1972) 179-198.

[55] RASKIND W., A finiteness theorem in the Galois cohomology of algebraic number fields, Trans. Amer. Math. Soc. 303 (1987) 743-749.

[56] Rost M., Chow groups with coefficients, Doc. Math. 1 (1996) 319-393.

[57] Soulé C., Groupes de Chow et $K$-théorie de variétés sur un corps fini, Math. Ann. 268 (1984) $317-$ 345 .

[58] SouLÉ C., Opérations en $K$-théorie algébrique, Can. Math. J. 37 (1985) 488-550. 
[59] SPIESS M., Proof of the Tate conjecture for products of elliptic curves over finite fields, Math. Ann. 314 (1999) 285-290.

[60] Suslin A., Algebraic $K$-theory of fields, in: Proceedings of the International Congress of Mathematicians, Berkeley, 1986, pp. 222-244.

[61] TATE J.T., Algebraic cycles and poles of zeta functions, in: Arithmetical Algebraic Geometry (Proc. Conf. Purdue Univ., 1963), 1965, pp. 93-110.

[62] TATE J.T., Endomorphisms of abelian varieties over finite fields, Invent. Math. 2 (1966) 134-144.

[63] TATE J.T., Conjectures on algebraic cycles in $l$-adic cohomology, in: Motives, in: Proc. Symposia Pure Math., vol. 55(1), Amer. Math. Society, Providence, RI, 1994, pp. 71-83.

[64] Voevodsky V., Motivic cohomology with $\mathbf{Z} / 2$ coefficients, Publ. Math. IHÉS, à paraître.

[65] WeIL A., Courbes algébriques et variétés abéliennes, Hermann, 1954, rééd. 1971.

[66] ZARhin Y.G., Abelian varieties of K3 type, in: Séminaire de théorie des nombres, Paris, 1990-1991, in: Progr. Math., vol. 108, Birkhäuser, 1992, pp. 263-279.

(Manuscrit reçu le 22 juillet 2002; accepté, après révision, le 14 février 2003.)

\author{
Bruno KAHN \\ Institut de Mathématiques de Jussieu, \\ 175-179 rue du Chevaleret, \\ 75013 Paris, France \\ E-mail : kahn@math.jussieu.fr
}

